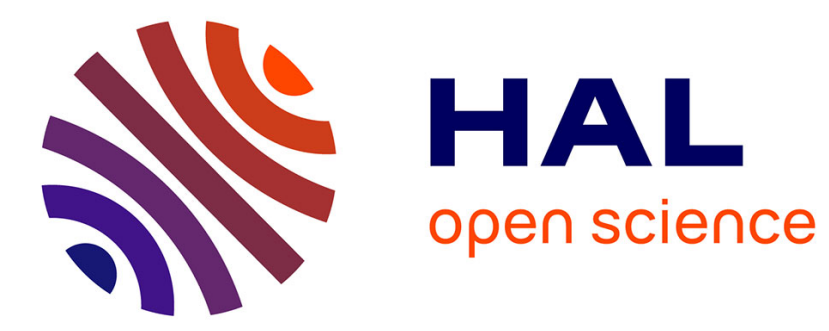

\title{
THE PREDICTABILITY OF \\ ADVECTION-DOMINATED FLUX-TRANSPORT SOLAR DYNAMO MODELS
}

Sabrina Sanchez, Alexandre Fournier, Julien Aubert

\section{- To cite this version:}

Sabrina Sanchez, Alexandre Fournier, Julien Aubert. THE PREDICTABILITY OF ADVECTIONDOMINATED FLUX-TRANSPORT SOLAR DYNAMO MODELS. The Astrophysical Journal, 2013, 781 (1), pp.8. 10.1088/0004-637X/781/1/8 . insu-01411576

\section{HAL Id: insu-01411576 \\ https://hal-insu.archives-ouvertes.fr/insu-01411576}

Submitted on 7 Dec 2016

HAL is a multi-disciplinary open access archive for the deposit and dissemination of scientific research documents, whether they are published or not. The documents may come from teaching and research institutions in France or abroad, or from public or private research centers.
L'archive ouverte pluridisciplinaire HAL, est destinée au dépôt et à la diffusion de documents scientifiques de niveau recherche, publiés ou non, émanant des établissements d'enseignement et de recherche français ou étrangers, des laboratoires publics ou privés. 


\title{
THE PREDICTABILITY OF ADVECTION-DOMINATED FLUX-TRANSPORT SOLAR DYNAMO MODELS
}

\author{
Sabrina Sanchez, Alexandre Fournier, and Julien Aubert \\ Institut de Physique du Globe de Paris, Sorbonne Paris Cité, Université Paris Diderot UMR 7154 CNRS, F-75005 Paris, France; ssanchez@ipgp.fr \\ Received 2013 May 30; accepted 2013 December 2; published 2013 December 24
}

\begin{abstract}
Space weather is a matter of practical importance in our modern society. Predictions of forecoming solar cycles mean amplitude and duration are currently being made based on flux-transport numerical models of the solar dynamo. Interested in the forecast horizon of such studies, we quantify the predictability window of a representative, advection-dominated, flux-transport dynamo model by investigating its sensitivity to initial conditions and control parameters through a perturbation analysis. We measure the rate associated with the exponential growth of an initial perturbation of the model trajectory, which yields a characteristic timescale known as the $e$-folding time $\tau_{e}$. The $e$-folding time is shown to decrease with the strength of the $\alpha$-effect, and to increase with the magnitude of the imposed meridional circulation. Comparing the $e$-folding time with the solar cycle periodicity, we obtain an average estimate for $\tau_{e}$ equal to 2.76 solar cycle durations. From a practical point of view, the perturbations analyzed in this work can be interpreted as uncertainties affecting either the observations or the physical model itself. After reviewing these, we discuss their implications for solar cycle prediction.
\end{abstract}

Key words: chaos - dynamo - Sun: activity

Online-only material: color figures

\section{INTRODUCTION}

The Sun is a magnetic active star, which undergoes successive phases of high and low magnetic activity with a quasi-periodicity of approximately $11 \mathrm{yr}$, powered by a natural dynamo mechanism (Moffatt 1978). This magnetic activity encompasses the recurrent manifestation of dynamical phenomena at the solar surface and in its atmosphere, such as sunspots, flares, and coronal mass ejections (Priest 1982). In addition to its remarkable regularity, solar activity exhibits longer term (decadal to centennial) fluctuations (Hathaway 2009), and occasional periods of long-lasting near-quiescence, such as the Maunder Minimum. Since the solar cycle affects the energy radiated by the Sun, its understanding is key in elucidating the potential control of solar activity on the long-term variability of the Earth's climate (Haigh 2003).

Solar activity influences the terrestrial environment in other important aspects, connected with the operation of satellites (Baker 2000), and the occurrence of geomagnetic storms, which can damage electric power grids and interfere with radars and radio communications. These important issues highlight the strong need for an accurate prediction of solar magnetic phenomena, which is one of the main goals of space weather (Pulkkinen 2007). Until recently, such forecasting exercises were mostly conducted within an entirely data-driven framework, based, for instance, on geomagnetic precursors methods (Hathaway 2009; Wang \& Sheeley 2009). It is sensible to believe, though, that more accurate and effective predictions could be obtained by combining these data with physical models of the Sun, using data assimilation (e.g., Talagrand 1997). The most salient illustration of the application of data assimilation emanates every day from numerical weather prediction (NWP) centers, in the form of weather forecasts (consult, e.g., Kalnay 2003 for a historical perspective on NWP). Application of data assimilation in geoscience also include oceanography (e.g., Brasseur 2006), the study of air quality (e.g., Elbern et al. 2010), and land surfaces (e.g., Houser et al. 2010). In a context similar to that of the solar dynamo, data assimilation has also recently come to the fore for the study of the Earth's dynamo, a surge motivated by our increased ability to observe and simulate the geomagnetic field (e.g., Fournier et al. 2007, 2010, 2013; Aubert \& Fournier 2011). Over the past $15 \mathrm{yr}$, the study of the solar dynamo has witnessed an even more spectacular increase in its observational and modeling capabilities. The question of the feasibility of applying data assimilation techniques to the solar dynamo was asked a few years ago (Brun 2007), and was followed by a series of studies bearing promises (Kitiashvili \& Kosovichev 2008; Jouve et al. 2011; Dikpati \& Anderson 2012).

The physical model of the solar dynamo that should enter this inverse problem machinery remains to be defined. Forward modeling of the solar dynamo has shed light on the main physical processes believed to be responsible for the solar cycle (see Charbonneau 2005 for a review). Kinematic dynamo theory stresses that these processes are connected with the continuous transformation of poloidal magnetic energy into toroidal magnetic energy (the $P \rightarrow T$ conversion), and vice versa (the $T \rightarrow P$ conversion, necessary to close the dynamo loop). There is now little doubt that the $\Omega$-effect, which denotes the shearing action of the differential rotation of the plasma flow, is responsible for the $P \rightarrow T$ conversion. Through the advent of helioseismology, the large-scale, interior, differential rotation was mapped in detail (Tomczyk et al. 1995), which made it possible to infer that the most likely location of the $\Omega$-effect is the base the convection zone, a region known as the tachocline (Howe et al. 2000). There is less consensus regarding the processes at work behind the $T \rightarrow P$ conversion. The meanfield $\alpha$-effect (Parker 1955), and the Babcock-Leighton (BL) mechanism (Babcock 1961; Leighton 1969) are two commonly envisioned possibilities. The former rests on the large scale effect of small scale turbulent motions whose twisting action can transform a toroidal field line into a poloidal field line. The latter relies on empirical evidences of the process of diffusion and reconfiguration of the magnetic field of sunspots. The three aforementioned processes ( $\alpha$-effect, $\Omega$-effect, and BL mechanism) are illustrated in Figure 1.

The ambiguity between the $\alpha$-effect scenario and the BL mechanism would disappear, should one be in a position to carry out the full three-dimensional numerical integration 


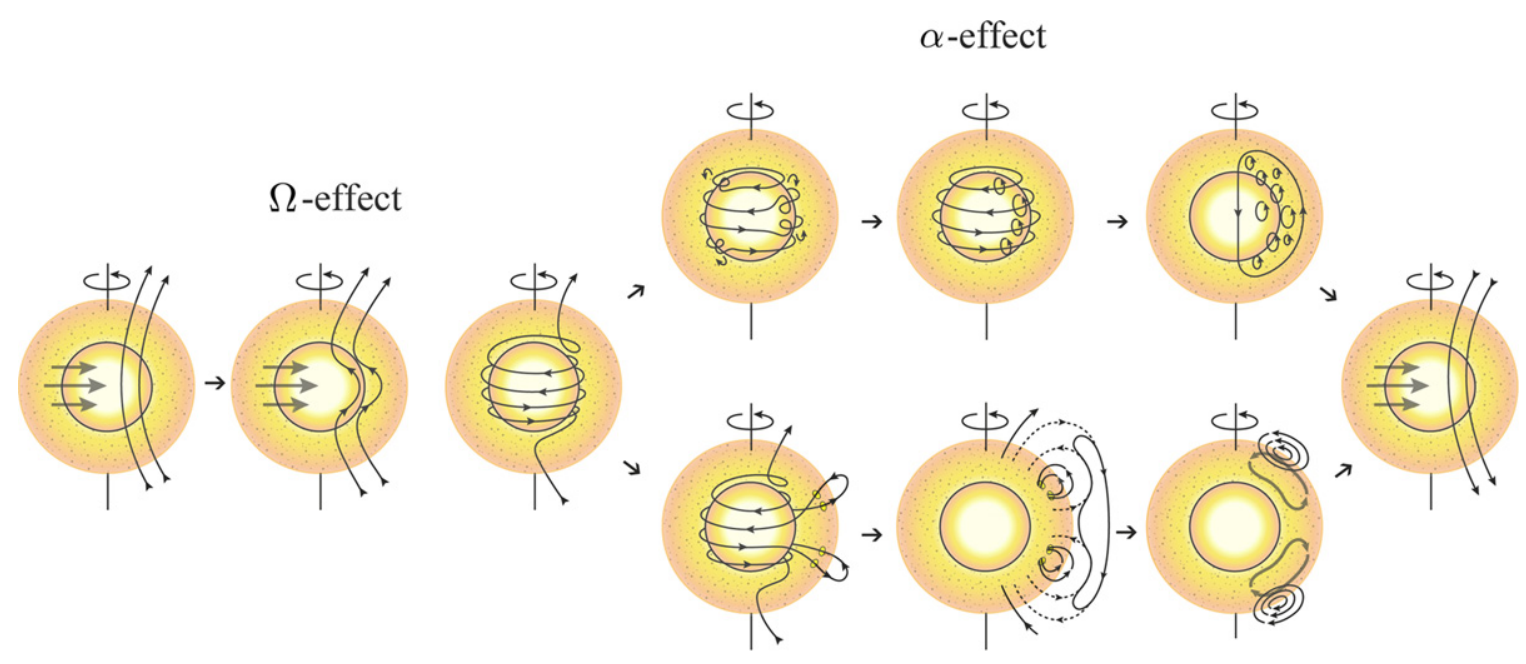

BL mechanism

Figure 1. Illustration of the main processes at work in our solar dynamo model. The $\Omega$-effect (left) depicts the transformation of a primary poloidal field into a toroidal field by means of the differential rotation. The poloidal field regeneration is next accomplished either by the $\alpha$-effect (top) and/or by the Babcock-Leighton mechanism (bottom). In the $\alpha$-effect case, the toroidal field at the base of the convection zone is subject to cyclonic turbulence. Secondary small-scale poloidal fields are thereby created, and produce on average a new, large-scale, poloidal field. In the Babcock-Leighton mechanism, the primary process for poloidal field regeneration is the formation of sunspots at the solar surface from the rise of buoyant toroidal magnetic flux tubes from the base of the convection zone. The magnetic fields of those sunspots nearest to the equator in each hemisphere diffuse and reconnect, while the field due to those sunspots closer to the poles has a polarity opposite to the current one, which initiates a polarity reversal. The newly formed polar magnetic flux is transported by the meridional flow to the deeper layers of the convection zone, thereby creating a new large-scale poloidal field.

(A color version of this figure is available in the online journal.)

of those equations governing the solar dynamo. Despite the monotonic and dramatic increase in compute power which already led to substantial achievements (e.g., Brun et al. 2004; Charbonneau \& Smolarkiewicz 2013), such a comprehensive integration remains out of reach due to the wide range of temporal and spatial scales induced by the high level of turbulence expected inside the solar convection zone. On the other hand, and from a more practical perspective, a large body of work has shown that axisymmetric mean-field solar dynamo models were able to reproduce many of the observed features of solar activity (Charbonneau 2005). The most recent and representative illustrations of this strand rely on the advection of magnetic flux by a meridional flow (following in general the BL mechanism). These models, called "flux-transport" models, are in particular successful in accounting for the equatorward migration of the solar toroidal field and the observed phaselocking of the solar cycle (Dikpati \& Charbonneau 1999; Charbonneau \& Dikpati 2000).

Such flux-transport models may make it possible to predict the amplitude and duration of the upcoming solar cycles. The first studies addressing this possibility (Dikpati et al. 2006; Choudhuri et al. 2007) considered direct incorporation of data into models, essentially by imposing (in a strong sense) surface boundary values inherited from the data onto the model, whereas an assimilation scheme would require this to happen in a weak sense, through some flavor of the so-called best linear unbiased estimator, whose goal is to combine in an optimal fashion the data and the model, considering the uncertainties affecting both. Independently of the data assimilation scheme one may resort to, and as good as it may be, there exists an intrinsic limit to its predictive power. Bushby \& Tobias (2007) point out that this limit arises either from the stochastic nature of the BL and $\alpha$-effects, or from nonlinear deterministic processes. They stress, in addition, that the lack of constraints on the exact nature of the key physical mechanisms which sustain these models and govern their time-dependency, such as the $\alpha$-effect, make their ability to capture the essentials of the solar dynamo process questionable. They conclude that under the best circumstances of a near-perfect model, the shape of the solar cycle could only be predicted one or two cycles ahead. As this best case scenario is out of reach, they argue that a reliable forecasting exercise is untractable.

The same critic was made regarding weather prediction during its early years. The seminal work by Lorenz (1963) showed the extreme sensitivity of a deterministic system governed by a simple set of nonlinear coupled differential equations to its initial conditions. In a subsequent study, Lorenz (1965) estimated the timescale of divergence $\tau$ of two initially very close dynamical trajectories (called twin trajectories in the following) to be of a few days (Lorenz's simple model aimed at representing atmospheric convection). More realistic models of the atmosphere have now established that $\tau$ is equal to two weeks. This value has to be confronted with the current forecast horizon of NWP, which is (depending on the center) between seven and nine days. The combined progress of observation, models, and data assimilation algorithms over the past $30 \mathrm{yr}$ has resulted roughly in a gain of one day per decade, bringing the operational limit closer and closer to the theoretical limit.

One may wonder to which extent the progress made by the atmospheric community could be expected within the solar community. Doing so, one immediately realizes that these two dynamical systems (the atmosphere and the Sun) are dramatically different. Whereas the Earth's atmosphere is a thin and directly observable layer, the solar convection zone is an almost entirely concealed thick shell. Moreover, the physics of the atmosphere is much better constrained than that at work behind the solar dynamo (consult Vallis 2006 for a review of atmospheric processes). Bearing these substantial differences in mind, and assuming that the basic physics involved in the solar dynamo is faithfully captured by mean-field models, one may 

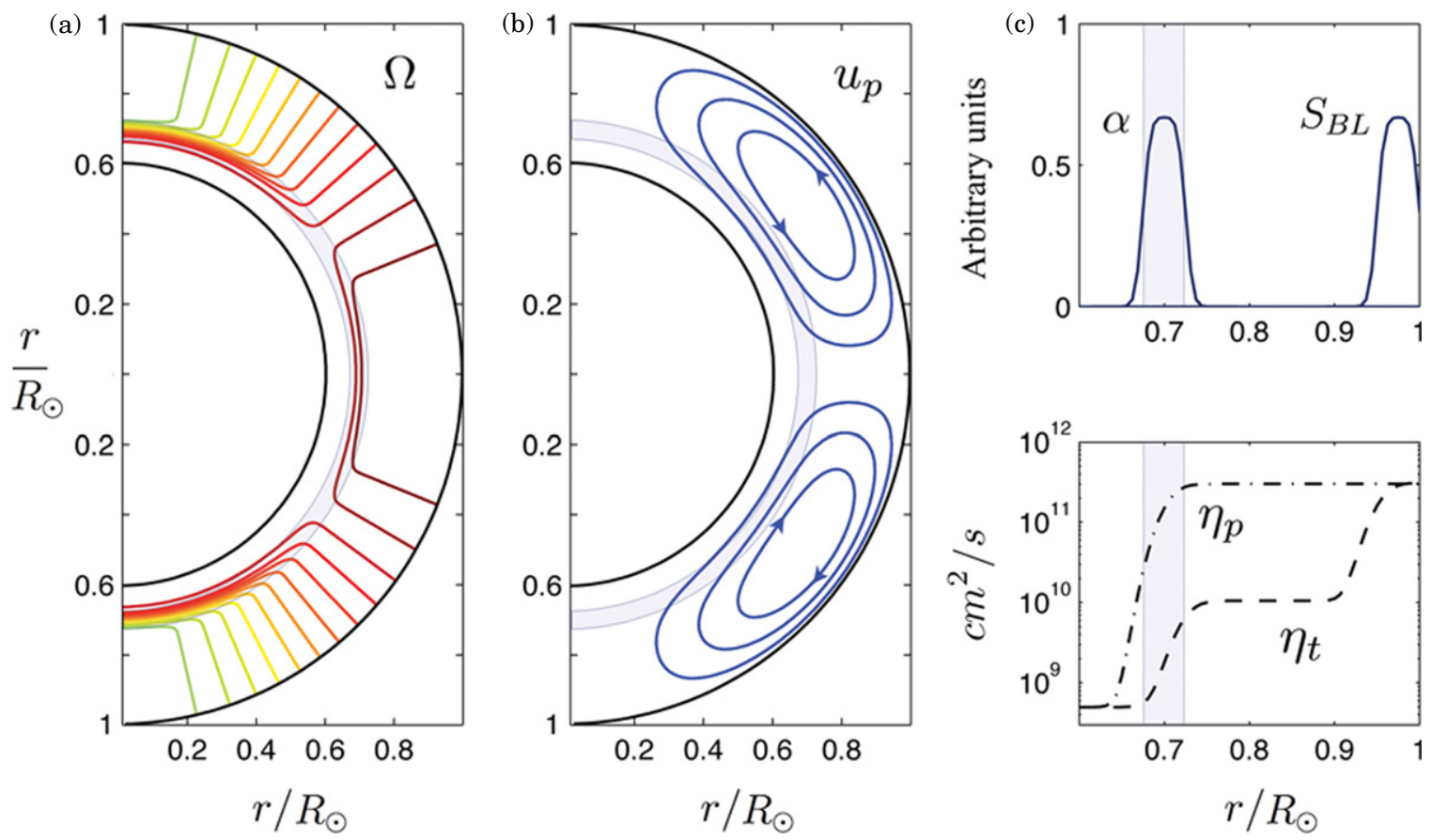

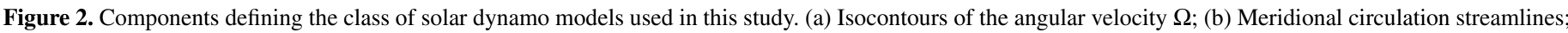

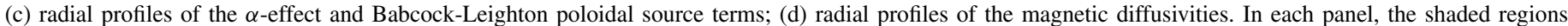

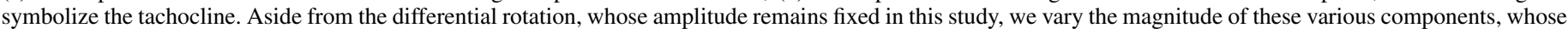
relative contributions are described by a suite of non-dimensional numbers (see the text for details).

(A color version of this figure is available in the online journal.)

still hope that the short-term prediction of at least some of the features of the solar cycle (e.g., duration and mean amplitude) is possible.

Knowledge of the modulations and mean intensity of the upcoming solar cycles from mean-field models may serve as an important input for more specific space weather considerations. In this study, we therefore wish to adopt an operational perspective. Assuming that mean-field models will be effectively used to forecast solar activity, our goal here is to quantify their intrinsic limit of predictability $\tau$ (the equivalent of the two weeks discussed above for the atmosphere), following the methodology proposed recently by Hulot et al. (2010) and Lhuillier et al. (2011) in order to estimate $\tau$ for the Earth's dynamo.

This paper is organized as follows. In Section 2, we describe our working mean-field model and detail its numerical implementation. We next inspect the sensitivity of this model to its control parameters in Section 3. Section 4 presents the systematic study of the error growth between twin trajectories. This allows us to evaluate $\tau$, and to assess its sensitivity to its control parameters. Finally, we discuss in Section 5 the influence of modeling and observational errors on the practical limit of predictability of the model.

\section{THE MODEL AND ITS NUMERICAL IMPLEMENTATION}

Our flux-transport model is the one presented by Sanchez et al. (2014); it includes both the $\alpha$ and BL scenarios for the $T \rightarrow P$ conversion. The first reason for adding an $\alpha$-effect to a standard $\mathrm{BL}$ flux-transport model is that a dynamo running on a $\mathrm{BL}$ mechanism alone cannot recover from a quiescent phase devoid of sunspots. As reported by Sanchez et al. (2014), the model setup enables the appearance of a long-term variability (succession of active and quiet phases), which can then be interpreted as the result of the competition between the $\alpha$-effect operating at the tachocline and a BL mechanism operating at the solar surface. In addition, a deep location of the $\alpha$-effect is known to favor the sought antisymmetrical evolution of the magnetic field in the Northern and Southern hemispheres (Dikpati \& Gilman 2001; Bonanno et al. 2002).

Let us now write accordingly the modified mean-field induction equation (Moffatt 1978) for the large-scale magnetic field $\mathbf{B}$

$$
\frac{\partial \mathbf{B}}{\partial t}=\nabla \times\left[\mathbf{U} \times \mathbf{B}-\eta \nabla \times \mathbf{B}+\alpha \mathbf{B}+S_{\mathrm{BL}} B_{\varphi} \hat{\mathbf{e}}_{\varphi}\right],
$$

where $\mathbf{U}$ is the prescribed flow, $\eta$ is the turbulent diffusivity, $\alpha$ is the turbulent magnetic helicity, and $S_{\mathrm{BL}} B_{\varphi} \hat{\mathbf{e}}_{\varphi}$ is the BL source term ( $\hat{\mathbf{e}}_{\varphi}$ is the unit vector in the direction of longitude). We will specify the profiles of these various physical fields in the following. The definitions that we will need are summarized in Table 1 and the profiles shown in Figure 2.

Under the assumption of axisymmetry, the magnetic and flow fields are further expressed in terms of their poloidal and toroidal components in spherical coordinates $(r, \theta, \varphi)$ as

$$
\begin{gathered}
\mathbf{B}(\mathbf{r}, \mathbf{t})=\nabla \times\left[A_{\varphi}(\mathbf{r}, t) \hat{\mathbf{e}}_{\varphi}\right]+B_{\varphi}(\mathbf{r}, t) \hat{\mathbf{e}}_{\varphi}, \\
\mathbf{U}(\mathbf{r})=\mathbf{u}_{p}(\mathbf{r})+r \sin \theta \Omega(\mathbf{r}) \hat{\mathbf{e}}_{\varphi},
\end{gathered}
$$

in which $A_{\varphi}$ is the poloidal potential and $B_{\varphi}$ is the toroidal field. The prescribed time-independent flow is defined by the angular velocity $\Omega$ and the meridional circulation $\mathbf{u}_{p}$, shown in Figures 2(a) and (b), respectively. Helioseismic data provide strong constraints on $\Omega$, which will thus remain fixed in the remainder of this work, and approximated using the analytic formula of Dikpati \& Charbonneau (1999). On the 
Table 1

Summary of the Mathematical Symbols Used in the Model, Their Values and a Brief Explanation of Their Meaning

\begin{tabular}{lcl}
\hline \hline Symbol & Value & \multicolumn{1}{c}{ Interpretation } \\
\hline$r_{t c}$ & $0.7 R_{\odot}$ & Radial location of the center of the tachocline \\
$\delta r$ & $0.05 R_{\odot}$ & Thickness of the tachocline \\
$\Omega_{\mathrm{eq}}$ & $2 \pi \times 460.7 \mathrm{nHz}$ & Rotation rate at the equator \\
$\alpha_{0}$ & $0.34-1.03 \mathrm{~m} \mathrm{~s}^{-1}$ & Strength of the $\alpha$-effect \\
$S_{\mathrm{BL}}$ & $0.02-0.06 \mathrm{~m} \mathrm{~s}^{-1}$ & Strength of the Babcock-Leighton mechanism \\
$u_{0}$ & $13.27-17.68 \mathrm{~m} \mathrm{~s}^{-1}$ & Velocity of the superficial meridional flow at mid-latitude \\
$\eta_{r}$ & $5 \times 10^{8} \mathrm{~cm}^{2} \mathrm{~s}^{-1}$ & Effective diffusivity near the radiative zone \\
$\eta_{c z}$ & $1 \times 10^{10} \mathrm{~cm}^{2} \mathrm{~s}^{-1}$ & Effective diffusivity at the bottom of the convection zone \\
$\eta_{s}$ & $3 \times 10^{11} \mathrm{~cm}^{2} \mathrm{~s}^{-1}$ & Effective diffusivity at the solar surface \\
\hline
\end{tabular}

contrary, the large-scale meridional circulation $\mathbf{u}_{p}$ remains poorly constrained. For the sake of simplicity, we will follow the one-cell per hemisphere description of Dikpati \& Charbonneau (1999).

The poloidal-toroidal decomposition of the magnetic and flow fields prompts us to define poloidal and toroidal components for the turbulent diffusivity $\eta$, denoted by $\eta_{p}$ and $\eta_{t}$, respectively. This distinction rests on the analysis made by Chatterjee et al. (2004), who pointed out that the toroidal field strength is expected to be much larger than the poloidal field strength throughout the convection zone. This should decrease notably the efficiency of toroidal turbulent diffusion compared with its poloidal counterpart. With this distinction at hand, injection of Equations (2) and (3) into Equation (1) gives rise to a set of two coupled partial differential equations for $A_{\varphi}$ and $B_{\varphi}$

$$
\begin{aligned}
& \frac{\partial A_{\varphi}}{\partial t}+\frac{\mathbf{u}_{p}}{r \sin \theta} \cdot \nabla\left(r \sin \theta A_{\varphi}\right)= \eta_{p}\left(\nabla^{2}-\frac{1}{r^{2} \sin ^{2} \theta}\right) A_{\varphi} \\
&+\alpha\left(r, \theta ; B_{\varphi}\right) B_{\varphi} \\
&+S_{\mathrm{BL}}\left(r, \theta ; B_{\varphi}^{t c}\right) B_{\varphi}^{t c}, \\
& \frac{\partial B_{\varphi}}{\partial t}+r \sin \theta \nabla \cdot\left(\frac{\mathbf{u}_{p} B_{\varphi}}{r \sin \theta}\right)= \eta_{t}\left(\nabla^{2}-\frac{1}{r^{2} \sin ^{2} \theta}\right) B_{\varphi} \\
&+\frac{1}{r} \frac{\partial \eta_{t}}{\partial r} \frac{\partial\left(r B_{\varphi}\right)}{\partial r} \\
&+r \\
& \sin \theta\left(\nabla \times A_{\varphi} \hat{\mathbf{e}}_{\varphi}\right) \cdot(\nabla \Omega),
\end{aligned}
$$

where $B_{\varphi}^{t c}=B_{\varphi}\left(r=r_{t c}, \theta, t\right)$ is the toroidal field at the tachocline, defined in this work as the spherical shell of mean radius $r_{t c}=0.7 R_{\odot}$, with a thickness $\delta r=0.05 R_{\odot}$. The dependency of the $S_{\mathrm{BL}}$ term in Equation (4) expresses the non-local character of the BL source term. Even if it is active within the surface layers, the BL regeneration process is thought to originate from processes occurring in the vicinity of the tachocline-numerical models indeed indicate that the formation of tilted bipolar regions at the surface is mostly controlled by the strength of toroidal flux tubes prior to their buoyant instability (D'Silva \& Choudhuri 1993). Their finite rise time should induce a time lag between the onset of the instability and the formation of the bipolar regions, on the order of some days to a few weeks (Jouve et al. 2010). We will neglect this delay on the account of it being small compared to the timescales of interest here.
Turning now our attention to the $\alpha$-effect, we use the standard formula of $\alpha$-quenching, which is written as

$$
\alpha\left(\mathbf{r} ; B_{\varphi}\right)=\frac{\alpha_{0}}{1+\left(\frac{B_{\varphi}}{B_{\text {eq }}}\right)^{2}} f_{\alpha}(\mathbf{r}),
$$

in which $\alpha_{0}$ is a typical magnitude, $B_{\text {eq }}=10^{4} \mathrm{G}$ (Fan 2009) and $f_{\alpha}(\mathbf{r})$ restricts the $\alpha$-effect to the mid-latitudes of the tachocline, according to

$f_{\alpha}(\mathbf{r})=\frac{1}{4}\left[1+\operatorname{erf}\left(\frac{r-r_{1}}{d_{1}}\right)\right]\left[1-\operatorname{erf}\left(\frac{r-r_{2}}{d_{2}}\right)\right] \cos \theta \sin \theta$,

where $r_{1}=r_{t c}-\delta r / 2, r_{2}=r_{t c}+\delta r / 2$, and $d_{1}=d_{2}=0.01 R_{\odot}$. The radial variations of $f_{\alpha}$ are shown in Figure 2(c).

The BL $S_{\mathrm{BL}}$ source term operates within bounds of the magnetic field strength (D'Silva \& Choudhuri 1993), specifically between $B_{\varphi, \text { min }}^{t c}=10^{4} \mathrm{G}$ and $B_{\varphi, \max }^{t c}=10^{5} \mathrm{G}$. Denoting the magnitude of this source term by $S_{\mathrm{BL}_{0}}$, we write accordingly

$$
\begin{aligned}
S_{\mathrm{BL}}\left(\mathbf{r} ; B_{\varphi}^{t c}\right)= & \frac{S_{\mathrm{BL}_{0}}}{4}\left[1+\operatorname{erf}\left(B_{\varphi}^{t c 2}-B_{\varphi, \min }^{t c 2}\right)\right] \\
& \times\left[1-\operatorname{erf}\left(B_{\varphi}^{t c 2}-B_{\varphi, \text { max }}^{t c 2}\right)\right] f_{\mathrm{BL}}(\mathbf{r}) .
\end{aligned}
$$

The radial and latitudinal distribution $f_{\mathrm{BL}}(\mathbf{r})$ is in turn given by

$$
\begin{aligned}
f_{\mathrm{BL}}(\mathbf{r})= & \frac{1}{4}\left[1+\operatorname{erf}\left(\frac{r-r_{3}}{d_{3}}\right)\right] \\
& \times\left[1-\operatorname{erf}\left(\frac{r-r_{4}}{d_{4}}\right)\right] \cos \theta \sin \theta,
\end{aligned}
$$

where $r_{3}=0.95 R_{\odot}, r_{4}=R_{\odot}$ and $d_{3}=d_{4}=0.01 R_{\odot}$. The radial distribution of $f_{\mathrm{BL}}$ is shown in Figure 2(c).

The poloidal and toroidal diffusivities in Equations (4) and (5), are written as

$$
\begin{aligned}
\eta_{p}(r)= & \eta_{r}+\eta_{s} \frac{1}{2}\left[1+\operatorname{erf}\left(\frac{r-r_{5}}{d_{5}}\right)\right], \\
\eta_{t}(r)= & \eta_{r}+\eta_{c z} \frac{1}{2}\left[1+\operatorname{erf}\left(\frac{r-r_{6}}{d_{6}}\right)\right] \\
& +\eta_{s} \frac{1}{2}\left[1+\operatorname{erf}\left(\frac{r-r_{7}}{d_{7}}\right)\right],
\end{aligned}
$$

in which $r_{5}=0.7 R_{\odot}, r_{6}=0.72 R_{\odot}, r_{7}=0.95 R_{\odot}, d_{5}=$ $d_{6}=d_{7}=0.025 R_{\odot}, \eta_{r}$ is the diffusivity at the boundary 
with the radiative zone, $\eta_{c z}$ is the diffusivity in the turbulent convection zone, and $\eta_{s}$ is the diffusivity in the surface layers (which applies to the poloidal field over the entire convection zone). The radial profiles of the diffusivities are shown in Figure 2(d). This model pertains to the generic class of advection-dominated models: owing to the low values of the diffusivities throughout the convection zone, the coupling between the regions where the poloidal and toroidal fields are generated is ensured by the meridional circulation. In diffusiondominated models (e.g., Chatterjee et al. 2004), this coupling is on the contrary accomplished by turbulent diffusion.

In order to express the dynamo equations in their nondimensional form, we choose the solar radius $R_{\odot}$ as the length scale and the magnetic diffusion time $R_{\odot}^{2} / \eta_{s}$ as the timescale (roughly equal to $500 \mathrm{yr}$ ). This yields

$$
\begin{aligned}
\frac{\partial A_{\varphi}}{\partial t}+\frac{\mathrm{Rm}}{r \sin \theta} \tilde{\mathbf{u}}_{p} \cdot \nabla\left(r \sin \theta A_{\varphi}\right) & =\tilde{\eta}_{p}\left(\nabla^{2}-\frac{1}{r^{2} \sin ^{2} \theta}\right) A_{\varphi} \\
& +C_{\alpha} \tilde{\alpha} B_{\varphi}+C_{\mathrm{BL}} \tilde{S}_{\mathrm{BL}} B_{\varphi}^{t c},
\end{aligned}
$$

$$
\begin{aligned}
\frac{\partial B_{\varphi}}{\partial t}+\operatorname{Rm} r \sin \theta \boldsymbol{\nabla} \cdot\left(\frac{\tilde{\mathbf{u}}_{p} B_{\varphi}}{r \sin \theta}\right) & =\tilde{\eta}_{t}\left(\nabla^{2}-\frac{1}{r^{2} \sin ^{2} \theta}\right) B_{\varphi} \\
& +\frac{1}{r} \frac{\partial \tilde{\eta}_{t}}{\partial r} \frac{\partial\left(r B_{\varphi}\right)}{\partial r}+C_{\Omega} r \sin \theta \\
& \times\left(\nabla \times A_{\varphi} \hat{\mathbf{e}}_{\varphi}\right) \cdot(\nabla \tilde{\Omega}) . \quad(13)
\end{aligned}
$$

Equations (12) and (13) contain six nondimensional numbers characterizing the relative importance of each term in the equations

$$
\begin{gathered}
\mathrm{Rm}=u_{o} R_{\odot} / \eta_{s}, \\
C_{\Omega}=\Omega_{\mathrm{eq}} R_{\odot}^{2} / \eta_{s}, \\
C_{\alpha}=\alpha_{0} R_{\odot} / \eta_{s}, \\
C_{\mathrm{BL}}=S_{\mathrm{BL}_{0}} R_{\odot} / \eta_{s}, \\
\text { the ratio } \eta_{r} / \eta_{s}, \\
\text { and the ratio } \eta_{c z} / \eta_{s} .
\end{gathered}
$$

The magnetic Reynolds number $\mathrm{Rm}$ is associated with the amplitude of the large-scale meridional flow, $u_{0}$. The three following coefficients $C_{\Omega}, C_{\alpha}, C_{\mathrm{BL}}$, respectively, express the ratio of the equatorial rotation, turbulent and $\mathrm{BL}$ timescales to the diffusive timescale. In these expressions, $\Omega_{\mathrm{eq}}$ is the equatorial rotation rate, and $\alpha_{0}$ and $S_{\mathrm{BL}_{0}}$ are the amplitudes of the $\alpha$ and BL terms seen above. The remaining two terms $\eta_{r} / \eta_{s}$ and $\eta_{c z} / \eta_{s}$ are magnetic diffusivity ratios entering the nondimensional forms of Equations (10) and (11). The $\sim$ in Equations (12) and (13) denotes normalization with respect to those quantities. Note that a suitable rescaling of $A_{\varphi}$ can decrease the number of control parameters by one, as it can scale either $C_{\alpha}$ or $C_{\mathrm{BL}}$ out of the problem (it is the ratio of these two that would remain). Albeit more elegant, we did not consider this possibility. We shall therefore analyze the $\alpha$ and BL effects independently in the remainder of this study.
Finally, our formulation has to be complemented with boundary and initial conditions. The inner boundary condition is that of a perfect conductor. An approximation of this condition is that

$$
\begin{aligned}
A_{\varphi}=B_{\varphi} & =0 \text { at the inner radius } \\
r & =0.6 R_{\odot}(\text { Chatterjee et al. 2004). }
\end{aligned}
$$

The outer boundary condition corresponds to the interface with an insulating medium, and requires matching of the internal solar field with a potential field (Dikpati \& Charbonneau 1999).

As an initial condition, we choose a dipolar field confined inside the convection zone. In this case,

$$
\begin{gathered}
A_{\varphi}(\mathbf{r}, t=0)=\sin \theta / r^{2} \text { for } 0.7 R_{\odot} \leqslant r \leqslant R_{\odot}, \\
A_{\varphi}(\mathbf{r}, t=0)=0 \text { elsewhere } \\
B_{\varphi}(\mathbf{r}, t=0)=0 \text { everywhere. }
\end{gathered}
$$

The numerical approximation of the problem at hand is based on the Parody code, which was originally designed for three-dimensional geodynamo simulations (Dormy et al. 1998; Aubert et al. 2008), and successfully passed the dynamo benchmark of Christensen et al. (2001). The magnetic field is expanded according to the three-dimensional poloidal-toroidal decomposition

$$
\mathbf{B}=\nabla \times \nabla \times(\mathcal{P} \mathbf{r})+\nabla \times(\mathcal{T} \mathbf{r})
$$

where the poloidal and toroidal scalar potentials $\mathcal{P}$ and $\mathcal{T}$ are further expanded upon an axisymmetric spherical harmonic basis $Y_{n}^{0}(\theta)$, according to

$$
(\mathcal{P}, \mathcal{T})(r, \theta, t)=\sum_{n=1}^{N}\left(\mathcal{P}_{n}, \mathcal{T}_{n}\right)(r, t) Y_{n}^{0}(\theta),
$$

and truncated at spherical harmonic degree $N$. The discretization is completed by applying a second-order finite differencing in radius and second order time integration, comprising a CrankNicolson scheme for the diffusive terms and a second order Adams-Bashforth scheme for the nonlinear terms. The resulting code was then successfully tested against the reference solutions of Jouve et al. (2008). Details of this benchmark are provided in the Appendix. The results presented in what follows were obtained using $N=65$, and $N_{r}=65$ uniform radial levels in $\left[0.6 R_{\odot}, R_{\odot}\right]$, and a constant non-dimensional time step size $\Delta t=5 \times 10^{-6}$. A typical run comprised $10^{7}$ time steps, which corresponds roughly to $25,000 \mathrm{yr}$.

\section{FORWARD MODELING: MODEL PROPERTIES AND VARIABILITY}

With our operational purpose in mind, a representative solution of the model should match some of the basic solar cycle features (Charbonneau 2005): cyclic polarity reversals with approximately $11 \mathrm{yr}$ periodicity; strong toroidal fields at the base of the convection zone migrating from mid-latitudes toward the equator; poleward migration of a weaker high-latitude magnetic field; phase lag of $\pi / 2$ between the toroidal field at mid-latitudes and polar field at the poles; antisymmetry of the magnetic field between the northern and southern hemispheres; and long-term variability of the solar cycle. 


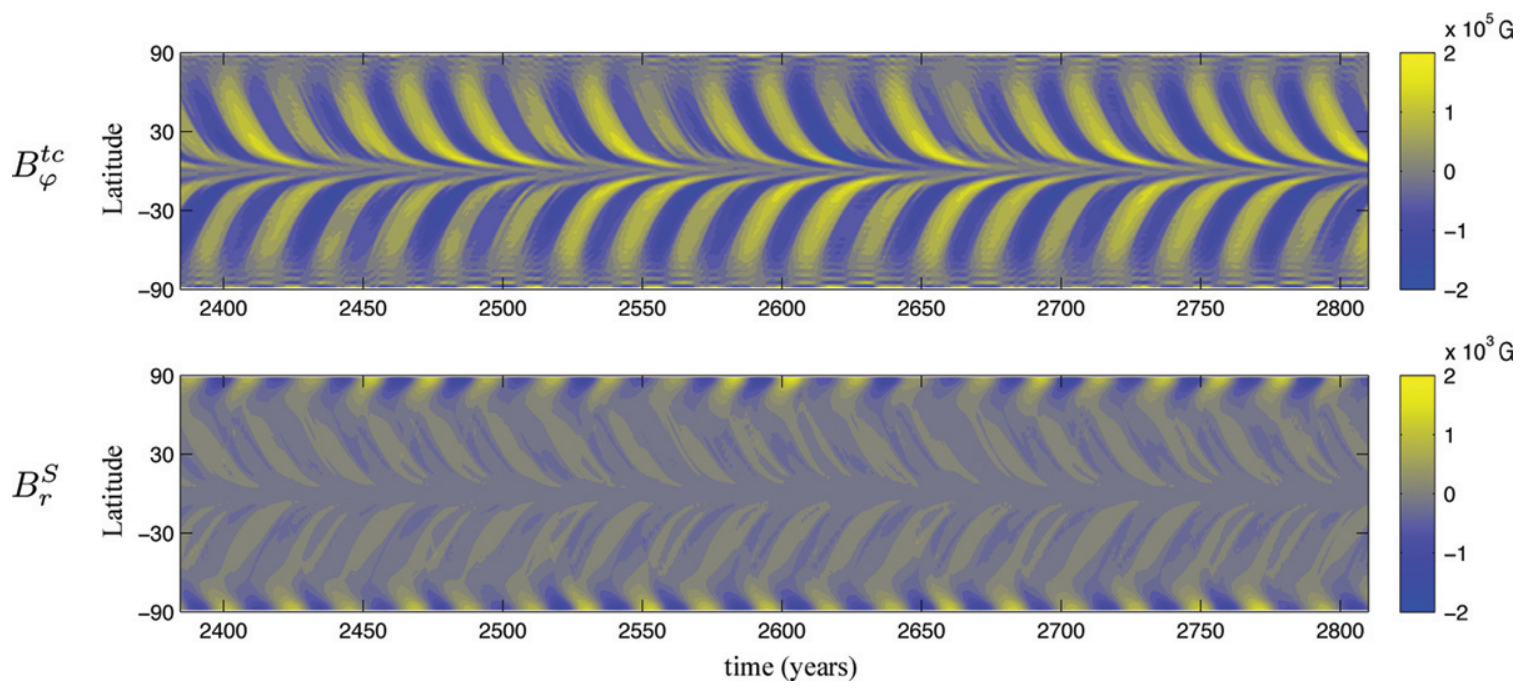

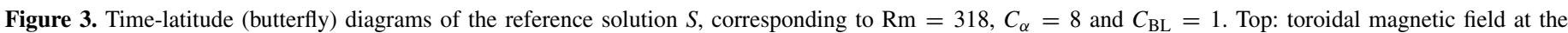
tachocline; bottom: radial magnetic field at the solar surface.

(A color version of this figure is available in the online journal.)

In the following, we will impose the fixity of some of those non-dimensional numbers appearing in Equations (14)-(19). As helioseismological data give $\Omega_{\mathrm{eq}} \sim 2 \pi \times 460.7 \mathrm{nHz}$, we set accordingly $C_{\Omega}=4.710^{4}$. In addition, the turbulent diffusivity in the solar interior is not well constrained (Ossendrijver 2003), and we consequently hold for simplicity the ratios $\eta_{r} / \eta_{s}$ and $\eta_{c z} / \eta_{s}$ fixed to values (see Table 1) previously shown to yield a satisfactory degree of solar semblance (e.g., Dikpati \& Charbonneau 1999). Variations of the remaining free parameters $C_{\alpha}, C_{\mathrm{BL}}$, and $\mathrm{Rm}$ allow for a broad range of solutions. Rm represents the strength of the meridional circulation and controls the periodicity of the solar cycle, a well-known characteristic of flux transport dynamos (Dikpati \& Charbonneau 1999). Consequently, and because of the strong observational constraint to obtain a period close to $11 \mathrm{yr}$, our family of models works in relatively narrow range of $\mathrm{Rm}$. As the meridional flow measured at the solar surface at mid-latitudes has an average magnitude $u_{0}$ of $15 \mathrm{~m} \mathrm{~s}^{-1}$ (Hathaway 1996), we vary Rm between 308 and 378 ( $u_{0} \approx 13$ and $17 \mathrm{~m} \mathrm{~s}^{-1}$, respectively). Within this range, getting a self-sustained reversing dynamo requires $C_{\alpha} \gtrsim 2$ and $C_{\mathrm{BL}} \gtrsim 0.5$.

We pick a reference (standard) solution (labeled $\mathrm{S}$ in the following) which has $\mathrm{Rm}=318, C_{\alpha}=8$ and $C_{\mathrm{BL}}=1$; it generates quasi-periodic reversals, separated by approximately 10.95 yr. Figure 3 represents the simulated evolutions of the toroidal field at the tachocline, $B_{\phi}^{t c}$, and of the radial field at the surface, $B_{r}^{S}$. It illustrates that the criteria for solar semblance which we listed are essentially met. This does not include the equatorial antisymmetric field configuration, a known recurring issue with BL models (Chatterjee et al. 2004; Charbonneau 2005). In this respect, Dikpati \& Gilman (2001) and Bonanno et al. (2002) previously showed that the addition of an $\alpha$-effect in a thin layer above the tachocline (as done here, recall Figure 2(c)) helps in obtaining antisymmetric solutions. However, and even if the portion of the dynamical trajectory represented in Figure 3 does display an antisymmetric magnetic field configuration, let us stress that there does not seem to exist a clear preferred mode of operation for the magnetic field: periods of symmetric, antisymmetric, and out-of-phase modes alternate over the dynamical trajectory followed by the standard model.

Long-term variability of the solar cycle is also present in this reference solution. Charbonneau et al. (2005) point out that chaotic modulation is a characteristic of BL models in which the BL term includes a lower operational threshold, as is the case in our model. Short periods of weaker than average activity level, lasting for approximately three cycles, are frequently found in our simulations, over a vast range of input parameters. In addition to this short-term variability, some of the solutions we obtain (including the reference solution $\mathrm{S}$ ) display as well long periods of grand minima, lasting for several centuries, during which the cycle is not fully developed, but persists with a residual activity (see Sanchez et al. 2014 for more details). The occurrence of long periods of minimum activity is rare in our simulations; we chose accordingly to focus on their regular, quasi-cyclic behavior to carry out the predictability analysis exposed below.

The quantities $C_{\alpha}$ and $C_{\mathrm{BL}}$ (recall their definition in Equations (16) and (17)) are less tightly constrained by observations than Rm, and they will constitute the effective degrees of freedom of our class of models when we investigate its horizon of predictability in the following section. Variations in $C_{\alpha}$ and $C_{\mathrm{BL}}$ affect the overall morphology of the solar cycle in different ways. While an increase in $C_{\alpha}$ tends to excite higher frequencies during the solar cycle, it does not result in strong alterations of the magnetic field strength and cycle periodicity. On the other hand, the intensity of the magnetic field is strongly and irregularly sensitive to variations of $C_{\mathrm{BL}}$ - the overall trend is that it grows with $C_{\mathrm{BL}}$. Increasing $C_{\mathrm{BL}}$ also usually results in the appearance of a feature respecting the Gnevyshev-Ohl rule, which is the persistent pattern of alternating high and low amplitudes of the solar cycles (Hathaway 2010). A too large an increase, though, gives rise to intermittent, non-solar-like, solutions. This forces us to define an upper bound of 2 for any admissible $C_{\mathrm{BL}}$. On the other hand, as the main role of $\mathrm{Rm}$ is that of setting the pace of the solar cycle, increasing its value leads to a shortening 
of the simulated periodicity (note that the first columns of Table 3, which we will discuss further below, document in detail this variability).

\section{PREDICTABILITY ANALYSIS}

\subsection{Methodology}

Our mean-field solar dynamo model is a dynamical system, characterized by a limited range of predictability, owing to its chaotic nature (Lorenz 1963). As stated in the Introduction, two initially very close, twin, dynamical trajectories are bound to diverge in a finite time $\tau$. The analysis of the divergence between these twin trajectories forms the backbone of our methodology; it is based on the work carried out by Hulot et al. (2010) and Lhuillier et al. (2011) to study the limit of predictability of the geodynamo.

We create a twin from a reference trajectory by perturbing a field variable (or control parameter) $\xi$ at a given instant $t_{p}$ in the following way

$$
\xi\left(t_{p}\right) \longmapsto \widetilde{\xi}\left(t_{p}\right)=\xi\left(t_{p}\right)(1+\varepsilon),
$$

where $\widetilde{\xi}$ and $\varepsilon$ are the perturbed quantity and the amplitude of the perturbation, respectively.

Of importance for the assessment of the predictability is the evolution of the distance between the two trajectories over time. In order to monitor this distance, we resort to two pointwise measures, which are related to the toroidal field $B_{\varphi}$ at a point $\mathbf{r}_{t c} \equiv\left(r=r_{t c}, \theta=70^{\circ}\right)$ on the tachocline, and to the radial field $B_{r}$ at a point $\mathbf{r}_{S} \equiv\left(r=R_{\odot}, \theta \sim 2^{\circ}\right)$ at the solar surface. These measures write

$$
\Delta B_{\varphi}\left(\mathbf{r}_{t c}, t\right) \equiv \frac{\left|B_{\varphi}\left(\mathbf{r}_{t c}, t\right)-\widetilde{B}_{\varphi}\left(\mathbf{r}_{t c}, t\right)\right|}{\sqrt{\left\langle B_{\varphi}^{2}\left(\mathbf{r}_{t c}\right)\right\rangle}}
$$

and

$$
\Delta B_{r}\left(\mathbf{r}_{S}, t\right) \equiv \frac{\left|B_{r}\left(\mathbf{r}_{S}, t\right)-\widetilde{B}_{r}\left(\mathbf{r}_{S}, t\right)\right|}{\sqrt{\left\langle B_{r}^{2}\left(\mathbf{r}_{S}\right)\right\rangle}},
$$

respectively. In these two definitions, notice that the distance is normalized since the brackets $\langle\cdot\rangle$ represent time averaging (which we perform over a period of about $1000 \mathrm{yr}$ after $t=t_{p}$ ). In the following, we will use $\Delta$ as a shorthand for $\Delta B_{\varphi}$ or $\Delta B_{r}$, when the distinction need not be made, and we will refer to the evolution of $\Delta$ as the error growth: in a forecasting perspective, the perturbation which we insert can indeed be interpreted as the uncertainty affecting the initial condition (or the control parameters) of the model. In this sense the distance we measure is analogous to the growth of the forecast error of interest for the data assimilation practitioner.

Figure 4 shows the typical evolution of the error growth (measured here in terms of $\Delta B_{\varphi}$ ) in our numerical experiments. It corresponds to a $\varepsilon=10^{-6}$ perturbation applied to the spectral poloidal coefficient $\mathcal{P}_{1}$. The evolution of $\Delta$ comprises three distinct phases. First, both trajectories remain fraternal, as their distance remain similar to $\varepsilon$ (phase I in Figure 4). This is called the mobilization phase by Lhuillier et al. (2011). Next, the error enters a phase of exponential growth (phase II), until it reaches saturation (phase III). From then on, the reference and perturbed solutions evolve in an uncorrelated way.

Among these three phases, the phase of exponential growth is the most meaningful to constrain the limit of predictability.
Considering that this phase starts at $t=t_{\varepsilon}$ with an initial value $\varepsilon$, the distance evolves according to

$$
\Delta(t)=\varepsilon e^{\lambda\left(t-t_{\varepsilon}\right)},
$$

where $\lambda$ denotes the exponential growth rate. Its inverse $\lambda^{-1}$ is the so-called $e$-folding time $\tau_{e}$, namely the divergence time $\tau$ we discussed above. We set out to estimate $\lambda$ (or $\tau_{e}$ ) as accurately as possible for the class of mean-field models considered in this work. Visual inspection of the time series of $\Delta$ allows us to pick the phase of exponential growth; we next perform a least-squares analysis to estimate $\lambda$ (this procedure yields the purple line in Figure 4).

That estimate may depend on the type and amplitude of the perturbation, though, which calls for a systematic approach to evaluating $\lambda$. In the next subsection, we use the standard model $S$ presented in Section 3 to vary extensively the type and amplitudes of perturbations. Within this single-model context, we find that the characteristics of the error growth are robust. Therefore, in order to push the analysis further, we shall consider in Section 4.3 how $\lambda$ may be influenced by the values of the triplet $\left(\mathrm{Rm}, C_{\mathrm{BL}}, C_{\alpha}\right)$.

\subsection{Error Growth in the Standard Model}

\subsubsection{Magnetic Perturbations}

As explained above, we focus here on the standard model $\mathrm{S}$ and begin by examining its response to perturbations of the magnetic field. We study different scenarios. The perturbation can affect either the poloidal scalar $\mathcal{P}$ or the toroidal scalar $\mathcal{T}$. It can be either large-scale (restricted to the $n=1$ harmonic degree), in which case it writes

$$
\mathcal{P}_{1}\left(r, t_{p}\right) \longmapsto \widetilde{\mathcal{P}_{1}}\left(r, t_{p}\right)=\mathcal{P}_{1}\left(r, t_{p}\right)(1+\varepsilon),
$$

(and the same for $\mathcal{T}_{1}$ ), or distributed randomly over the entire spectrum, according to

$$
\mathcal{P}_{n} \longmapsto \widetilde{\mathcal{P}_{n}}\left(r, t_{p}\right)=\mathcal{P}_{n}\left(r, t_{p}\right)\left(1+\gamma_{n} \varepsilon\right), 1 \leqslant n \leqslant N,
$$

(and the same for $\mathcal{T}_{n}$ ), in which the $\gamma_{n}$ are random numbers from 0 to 1 distributed over all the harmonic degrees. In the remainder of this subsection, the amplitude of the perturbation $\varepsilon$ is set to $10^{-6}$

The pink curves in Figure 5 show that large-scale perturbations of the poloidal or toroidal scalars defined by Equation (30) yield the same well-defined three phases for the evolution of $\Delta B_{r}$ and $\Delta B_{\varphi}$. In addition, each panel of Figure 5 comprises five gray curves obtained from five random realizations of the small-scale perturbations defined by Equation (31). Despite some scatter, visual inspection indicates a common error growth behavior. In particular, if we were to estimate $\lambda$ from this catalog of curves, we would probably get a robust value. This is rather encouraging, but before proceeding with the actual calculation of $\lambda$, let us now inspect in more detail its sensitivity to a broader range of perturbations.

\subsubsection{Systematic Perturbations}

We thus investigate now the error growth induced by perturbations of different origins, varying amplitudes $\varepsilon$ and different times of insertion $t_{p}$ on the standard model S. The origin of the perturbation $\xi$ in Equation (26) can be one of the following: $\mathcal{P}_{1}$, $\mathcal{T}_{1}, \mathcal{P}_{n}, \mathcal{T}_{n}$ (as in the previous section), $\Omega$ or $u_{p}$ (the flow), $\alpha$ or $S_{\mathrm{BL}}$ (the poloidal source terms), or $\eta_{p}$ (the poloidal diffusivity 

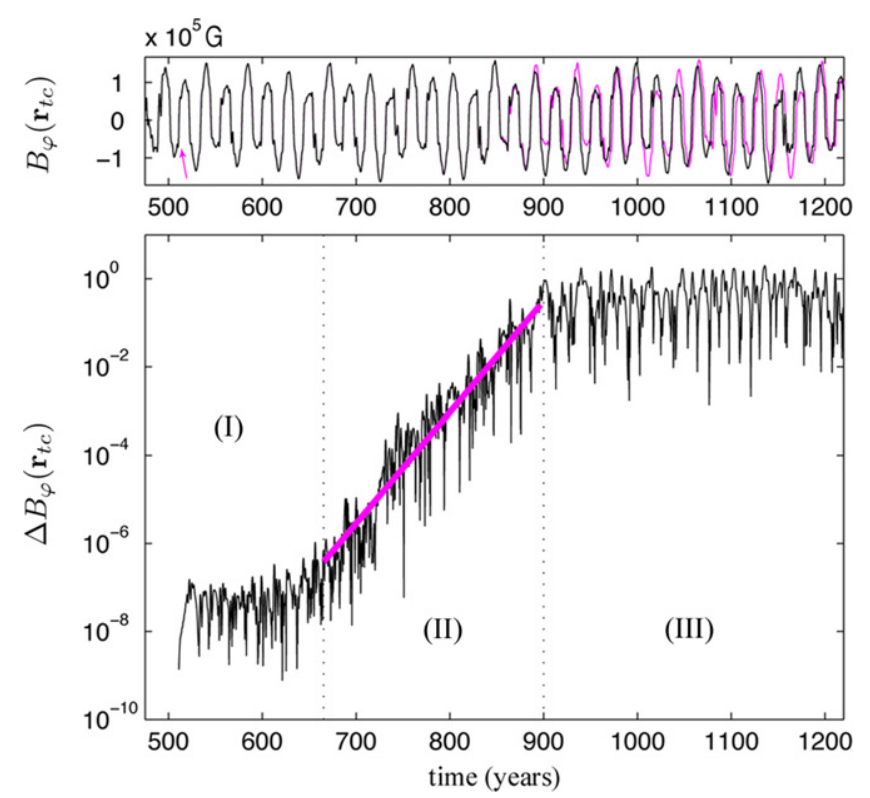

Figure 4. Error growth behavior for the reference model $\mathrm{S}$ (see Table 3). Top: toroidal field at $20^{\circ}$ latitude on the tachocline. The reference solution (shown in black) is perturbed at a given instant, shown by the pink arrow, by a relative amount $\varepsilon=10^{-6}$. This generates the perturbed solution (purple) which progressively diverges from the reference one. Bottom: the difference $\Delta B_{\varphi}$ between both solutions on a logarithmic scale. The error growth can be separated in three well-defined stages: (I) a mobilization phase, (II) an exponential growth phase and (III) a saturated phase. In phase (II), we perform a least-squares regression (purple line) in order to estimate the error growth rate $\lambda$. See the text for details.

profile). For each of these nine possibilities, we consider perturbations of amplitudes $10^{-2}, 10^{-4}, 10^{-6}, 10^{-8}$ or $10^{-10}$. Finally, we perturb the reference dynamical trajectory at three different times, $t_{p}=t_{1}, t_{2}$ or $t_{3}$. We therefore consider 9 (origins) $\times 5$ (amplitudes) $\times 3$ (instants) $=135$ ways of perturbing the standard trajectory. Since both $\Delta B_{\varphi}$ and $\Delta B_{r}$ are used to monitor the error growth, this allows us to construct a database of 270 estimates of $\lambda$. The database is completely described in Table 2 .

Figure 6 illustrates the variability within the database of model S, and shows that regardless of this variability, the error growth displays a fair amount of dynamical similarity in the 270 scenarios we envisioned. Figure 6(a) shows the evolution of $\Delta B_{\varphi}$, for different origins, times of perturbation insertion and different perturbation amplitudes. We see that the error growth is weakly sensitive to the origin of the perturbations. Still, the mobilization phase seems to vary depending on the way the perturbations were inserted. For perturbations corresponding to $\xi=\alpha$ or $\xi=u_{p}$, the mobilization phase lasts longer (several centuries), and there is a mild dependency of the duration of that phase on $t_{p}$. The mobilization phase has a duration which decreases with $\varepsilon$ as well. However, this variability on the mobilization phase does not strongly affect the estimate of $\lambda$. On another note, it can also be seen that the error growth due to smaller perturbations can experience secondary mobilization phases, and resume its exponential growth after some time.

Figure 6(b) presents the distribution of the error growth rates (one histogram per value of $\varepsilon$, which integrates all other dependencies) of model $\mathrm{S}$. The exponential growth is steeper for smaller levels of perturbations (noticing that $\varepsilon=10^{-8}$ and $\varepsilon=10^{-10}$ yield essentially the same behavior, though), that is, large perturbations lead to smaller values of $\lambda$. There is also a general tendency for the growth to slacken as the error reaches macroscopic values.

Still, focusing on small to extremely small values of $\varepsilon\left(10^{-6}\right.$ and less), the estimated $\lambda$ does not vary by more than $20 \%$. This robustness suggests the fact that $\lambda$ is an intrinsic property of our standard model $S$ : regardless of the perturbation time and origin, and as long as it is small, the exponential growth of the

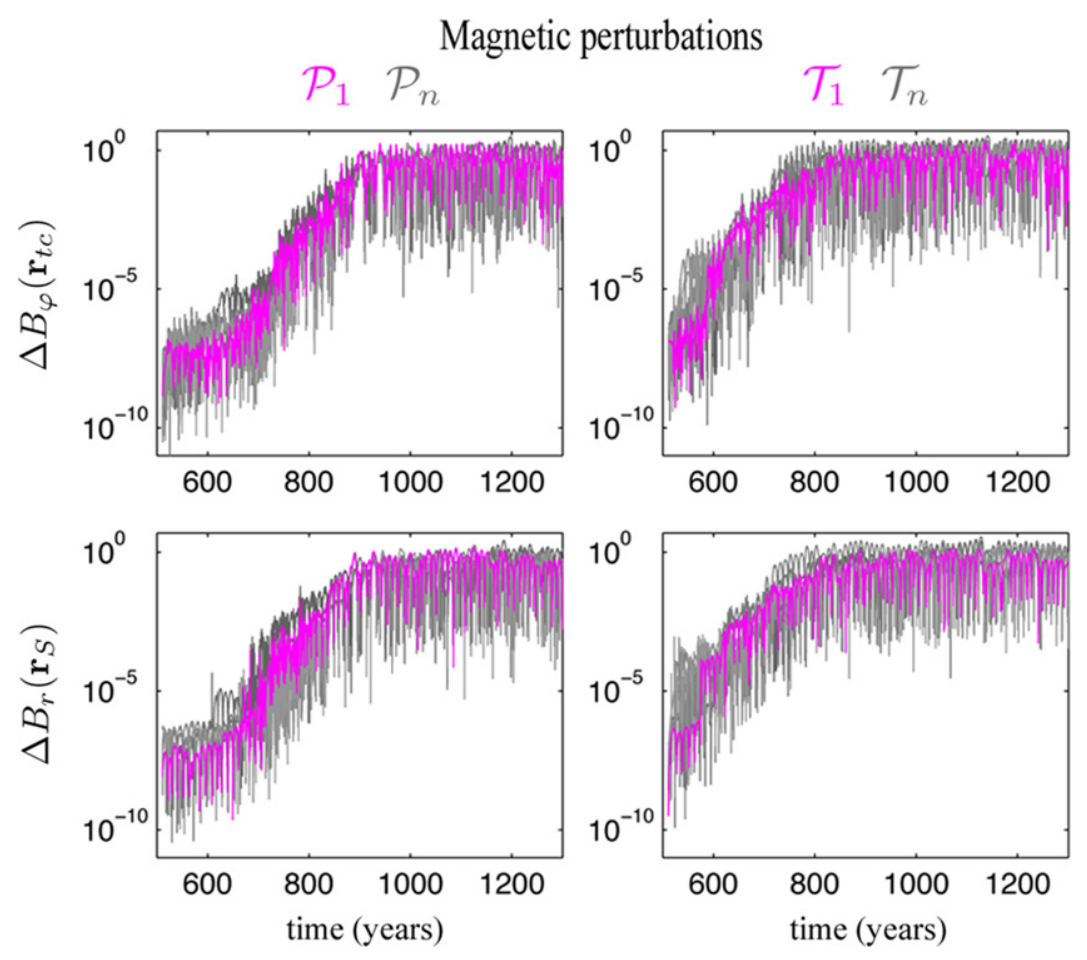

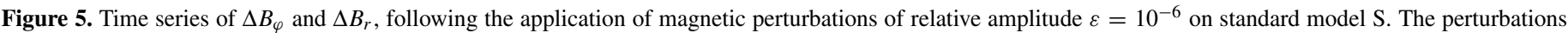

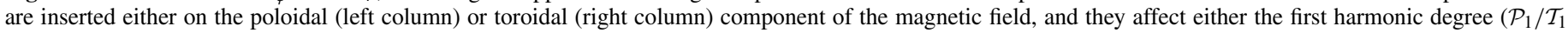
pink curves) or all the harmonic degrees randomly $\left(\mathcal{P}_{n} / \mathcal{T}_{n}\right)$, in which case five curves with different shades of gray are shown in each graph. 


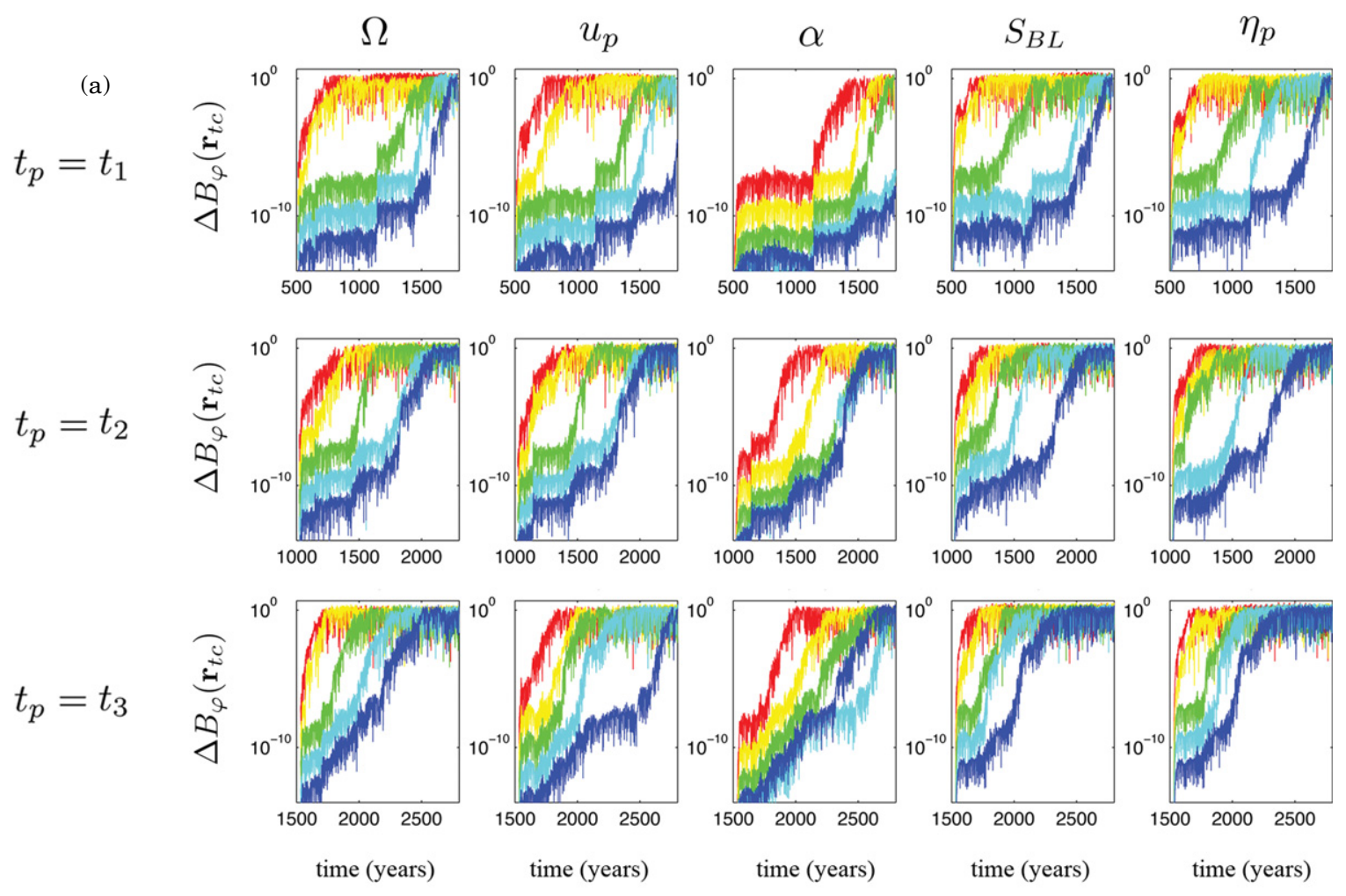

(b)
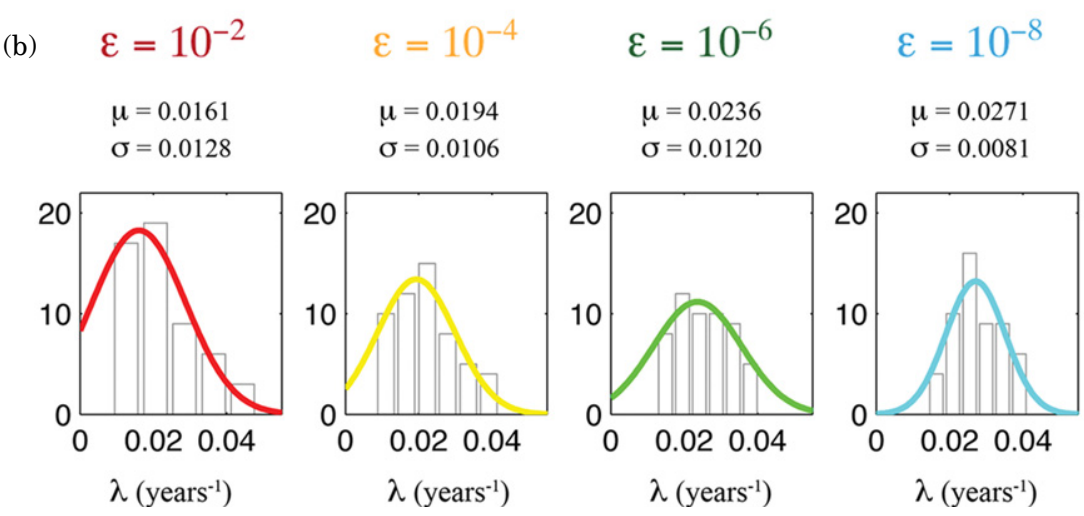

$$
\begin{aligned}
\varepsilon & =10^{-10} \\
\mu & =0.0273 \\
\sigma & =0.0033
\end{aligned}
$$

$\lambda\left(\right.$ years $\left.^{-1}\right)$

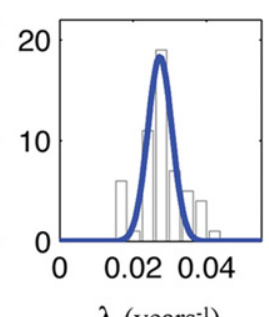

Figure 6. Sensitivity of the error growth rate against perturbation types for model S: (a) Error growth considering different origins $\left(\xi=\Omega, u_{p}, \alpha, S_{\mathrm{BL}}\right.$, and $\left.\eta_{p}\right)$, times $\left(t_{p}=t_{1}, t_{2}\right.$, and $\left.t_{3}\right)$ and amplitudes $\left(\varepsilon=10^{-2}, 10^{-4}, 10^{-6}, 10^{-8}\right.$, and $\left.10^{-10}\right)$; (b) histograms of the exponential growth rates $\lambda$ from the set of perturbations displayed in Table 2 ordered by perturbation amplitude. The histograms are modeled by Gaussian curves with mean $\mu$ and standard deviation $\sigma$.

error is likely to occur on a timescale $\tau$ of roughly $40 \mathrm{yr}$, that is over slightly more than three simulated cycles. More precisely, if $T_{c}$ denotes the period of the simulated cycle and considering a least-squares analysis of the $\varepsilon=10^{-10}$ histogram, we find that $\tau_{e}=(3.34 \pm 0.40) T_{c}$.

\subsection{Sensitivity of $\lambda$ to the Control Parameters}

We now explore the more general dependency of $\tau_{e}$ to the control parameters of our class of mean-field models. Since the simulated $T_{c}$ varies with these parameters as well, and since we wish to express $\tau_{e}$ in units of $T_{c}$, we investigate the joint dependency of these two quantities on the triplet (Rm, $\left.C_{\alpha}, C_{\mathrm{BL}}\right)$.

First, we increase the $\alpha$-effect coefficient from $C_{\alpha}=8$ to $C_{\alpha}=16$ and consider the same 270 possibilities as the ones used for the standard model (this new model is labeled $\mathrm{T}$ in the following). Figure 7 illustrates the corresponding database of model T, and highlights consistent differences when compared with the standard case S shown in Figure 6. Most notably, the mobilization phase is in every instance much shorter (not lasting more than a few decades), while the exponential growth phase is in all cases much steeper, two effects pointing toward an increased influence of turbulence as the value of $C_{\alpha}$ increases, leading to larger estimates for $\lambda$. We still retrieve the tendency for $\lambda$ to decrease with increasing $\varepsilon$, while its uncertainties decrease with $\varepsilon$. Accordingly, we find that for $\varepsilon=10^{-10}$, $\tau_{e}=(2.45 \pm 0.42) T_{c}$ (here, $T_{c}=10.15 \mathrm{yr}$ ).

Next, we carry out a similar sensitivity analysis with different triplets $\left(\mathrm{Rm}, C_{\alpha}, C_{\mathrm{BL}}\right)$. More specifically, we consider the following possibilities

1. $308 \leqslant \mathrm{Rm} \leqslant 378$,

2. $0.5 \leqslant C_{\mathrm{BL}} \leqslant 2$,

3. $8 \leqslant C_{\alpha} \leqslant 32$,

providing a total of 48 different models (including models $\mathrm{S}$ and T). For each model we calculate $\lambda$ restraining the amplitude of the perturbation $\varepsilon$ to what we consider its most reliable level, 
Table 2

Mean Values of $\lambda$ and Its Uncertainties $\sigma$ (in Units of $10^{-2} \mathrm{yr}^{-1}$ ) from the Systematic Perturbation Analysis of the Standard Model S

\begin{tabular}{|c|c|c|c|c|c|c|c|c|c|c|c|c|c|c|c|c|c|c|c|c|c|}
\hline \multirow[b]{3}{*}{$t_{p}$} & \multirow{3}{*}{$\begin{array}{l}\varepsilon \\
\xi\end{array}$} & \multicolumn{10}{|c|}{$\Delta B_{\varphi}$} & \multicolumn{10}{|c|}{$\Delta B_{r}$} \\
\hline & & \multicolumn{2}{|c|}{$10^{-10}$} & \multicolumn{2}{|c|}{$10^{-8}$} & \multicolumn{2}{|c|}{$10^{-6}$} & \multicolumn{2}{|c|}{$10^{-4}$} & \multicolumn{2}{|c|}{$10^{-2}$} & \multicolumn{2}{|c|}{$10^{-10}$} & \multicolumn{2}{|c|}{$10^{-8}$} & \multicolumn{2}{|c|}{$10^{-6}$} & \multicolumn{2}{|c|}{$10^{-4}$} & \multicolumn{2}{|c|}{$10^{-2}$} \\
\hline & & $\lambda$ & $\sigma$ & $\lambda$ & $\sigma$ & $\lambda$ & $\sigma$ & $\lambda$ & $\sigma$ & $\lambda$ & $\sigma$ & $\lambda$ & $\sigma$ & $\lambda$ & $\sigma$ & $\lambda$ & $\sigma$ & $\lambda$ & $\sigma$ & $\lambda$ & $\sigma$ \\
\hline \multirow[t]{9}{*}{$t_{1}$} & $\mathcal{P}_{1}$ & 3.66 & 0.07 & 2.49 & 0.05 & 2.52 & 0.06 & 1.68 & 0.09 & 3.57 & 0.21 & 3.69 & 0.09 & 2.33 & 0.06 & 2.58 & 0.06 & 1.44 & 0.09 & 4.23 & 0.35 \\
\hline & $\mathcal{T}_{1}$ & 3.62 & 0.07 & 3.20 & 0.13 & 1.52 & 0.05 & 1.33 & 0.07 & 3.82 & 0.35 & 3.70 & 0.09 & 3.51 & 0.11 & 1.51 & 0.05 & 1.60 & 0.09 & 1.19 & 0.22 \\
\hline & $\mathcal{P}_{n}$ & 3.48 & 0.06 & 1.49 & 0.06 & 1.87 & 0.08 & 1.21 & 0.08 & 2.76 & 0.27 & 3.69 & 0.08 & 1.51 & 0.05 & 2.05 & 0.06 & 1.33 & 0.07 & 1.21 & 0.21 \\
\hline & $\mathcal{T}_{n}$ & 2.89 & 0.06 & 2.12 & 0.08 & 1.86 & 0.11 & 2.43 & 0.21 & 1.61 & 0.15 & 3.04 & 0.06 & 2.11 & 0.07 & 1.89 & 0.10 & 2.29 & 0.14 & 1.52 & 0.13 \\
\hline & $\Omega$ & 3.16 & 0.05 & 3.82 & 0.09 & 2.30 & 0.06 & 1.40 & 0.05 & 2.11 & 0.09 & 3.98 & 0.10 & 3.52 & 0.11 & 2.90 & 0.08 & 2.22 & 0.06 & 2.20 & 0.14 \\
\hline & $u_{p}$ & 1.74 & 0.03 & 3.52 & 0.04 & 2.90 & 0.08 & 3.98 & 0.06 & 2.20 & 0.14 & 2.57 & 0.05 & 3.73 & 0.07 & 2.77 & 0.07 & 2.43 & 0.06 & 2.15 & 0.13 \\
\hline & $\alpha$ & 3.12 & 0.05 & 2.32 & 0.04 & 3.03 & 0.05 & 3.98 & 0.12 & 2.01 & 0.05 & 3.18 & 0.05 & 2.28 & 0.03 & 3.39 & 0.05 & 4.19 & 0.15 & 1.62 & 0.06 \\
\hline & $S_{\mathrm{BL}}$ & 2.73 & 0.05 & 3.39 & 0.08 & 1.92 & 0.04 & 1.91 & 0.13 & 4.83 & 0.25 & 2.91 & 0.05 & 3.58 & 0.09 & 1.84 & 0.05 & 1.67 & 0.09 & 2.18 & 0.14 \\
\hline & $\eta_{p}$ & 2.66 & 0.05 & 2.53 & 0.05 & 1.83 & 0.04 & 2.22 & 0.11 & 1.56 & 0.28 & 2.90 & 0.04 & 2.31 & 0.06 & 1.85 & 0.05 & 1.12 & 0.08 & 1.12 & 0.12 \\
\hline \multirow[t]{9}{*}{$t_{2}$} & $\mathcal{P}_{1}$ & 2.48 & 0.04 & 2.57 & 0.04 & 1.37 & 0.06 & 1.46 & 0.04 & 1.37 & 0.07 & 2.50 & 0.04 & 2.77 & 0.05 & 1.26 & 0.05 & 1.15 & 0.05 & 1.61 & 0.14 \\
\hline & $\mathcal{T}_{1}$ & 2.62 & 0.04 & 3.69 & 0.06 & 3.15 & 0.10 & 1.36 & 0.04 & 1. & 0.09 & 2.76 & 0.04 & 3.97 & 0.07 & 3.91 & 0.12 & 1.28 & 0.07 & 0.87 & 0.16 \\
\hline & $\mathcal{P}_{n}$ & 3.06 & 0.06 & 3.92 & 0.07 & 1.97 & 0.08 & 1.58 & 0.06 & 1.81 & 0.15 & 2.91 & 0.10 & 4.12 & 0.10 & 1.84 & 0.06 & .67 & 0.05 & 1.31 & 0.09 \\
\hline & $\mathcal{T}_{n}$ & 3.44 & 0.06 & 2.87 & 0.08 & 2.07 & 0.08 & 1.53 & 0.08 & 2.10 & 0.23 & 3.61 & 0.08 & 2.76 & 0.08 & 2.48 & 0.11 & 1.37 & 0.06 & 1.79 & 0.22 \\
\hline & $\Omega$ & 2.53 & 0.04 & 2.71 & 0.05 & 3.46 & 0.07 & 2.13 & 0.05 & 1.43 & 0.05 & 2.50 & 0.04 & 2.79 & 0.05 & 3.93 & 0.10 & 2.07 & 0.05 & 1.40 & 0.04 \\
\hline & $u_{p}$ & 2.68 & 0.04 & 2.80 & 0.05 & 3.47 & 0.05 & 2.16 & 0.11 & 1.96 & 0.09 & 2.67 & 0.04 & 2.54 & 0.04 & 3.60 & 0.06 & 1.32 & 0.05 & 2.16 & 0.10 \\
\hline & $\alpha$ & 3.11 & 0.05 & 2.81 & 0.05 & 4.03 & 0.04 & 2.65 & 0.05 & 3.35 & 0.09 & 3.19 & 0.05 & 2.83 & 0.06 & 2.31 & 0.04 & 3.07 & 0.05 & 2.57 & 0.11 \\
\hline & $S_{\mathrm{BL}}$ & 2.65 & 0.05 & 3.65 & 0.06 & 4.03 & 0.12 & 2.34 & 0.08 & 1.87 & 0.17 & 2.49 & 0.05 & 1.68 & 0.08 & 3.53 & 0.12 & 1.42 & 0.07 & 1.30 & 0.08 \\
\hline & $\eta_{p}$ & 2.47 & 0.04 & 3.15 & 0.04 & 1.98 & 0.12 & 2.22 & 0.08 & 1.78 & 0.08 & 2.57 & 0.06 & 1.42 & 0.05 & 1.38 & 0.04 & 1.42 & 0.09 & 1.98 & 0.14 \\
\hline \multirow[t]{9}{*}{$t_{3}$} & $\mathcal{P}_{1}$ & 2.37 & 0.04 & 2.32 & 0.03 & 2.85 & 0.05 & 2.05 & 0.12 & 2.53 & 0.21 & 2.75 & 0.0 & 2.26 & 0.03 & 2.76 & 0.05 & 2.94 & 0.20 & 3.06 & 0.35 \\
\hline & $\mathcal{T}_{1}$ & 2.59 & 0.05 & 2.57 & 0.07 & 3.19 & 0.07 & 2.45 & 0.16 & 1.37 & 0.32 & 2.69 & 0.06 & 2.07 & 0.06 & 2.61 & 0.08 & 3.65 & 0.25 & 1.84 & 0.22 \\
\hline & $\mathcal{P}_{n}$ & 4.40 & 0.12 & 2.48 & 0.05 & 2.73 & 0.14 & 0.82 & 0.33 & 2.00 & 0.29 & 2.73 & 0.06 & 2.47 & 0.05 & 2.45 & 0.12 & 2.52 & 0.20 & 2.06 & 0.25 \\
\hline & $\mathcal{T}_{n}$ & 1.64 & 0.02 & 2.81 & 0.06 & 2.81 & 0.10 & 3.07 & 0.19 & 2.72 & 0.32 & 1.96 & 0.04 & 2.59 & 0.05 & 2.48 & 0.10 & 3.16 & 0.24 & 3.65 & 0.34 \\
\hline & $\Omega$ & 1.50 & 0.01 & 1.87 & 0.03 & 3.07 & 0.08 & 2.74 & 0.18 & 1.98 & 0.14 & 1.54 & 0.01 & 1.91 & 0.03 & 3.29 & 0.07 & 2.65 & 0.13 & 2.82 & 0.22 \\
\hline & $u_{p}$ & 2.77 & 0.06 & 2.77 & 0.06 & 3.53 & 0.08 & 3.17 & 0.06 & 3.11 & 0.05 & 2.70 & 0.06 & 2.61 & 0.06 & 3.43 & 0.08 & 2.96 & 0.06 & 2.02 & 0.05 \\
\hline & $\alpha$ & 1.76 & 0.03 & 3.56 & 0.09 & 1.33 & 0.04 & 2.10 & 0.04 & 2.95 & 0.05 & 1.67 & 0.03 & 2.66 & 0.06 & 1.60 & 0.02 & 2.15 & 0.04 & 2.57 & 0.06 \\
\hline & $S_{\mathrm{BL}}$ & 2.42 & 0.08 & 2.44 & 0.07 & 2.80 & 0.05 & 2.84 & 0.18 & 4.31 & 0.35 & 2.64 & 0.06 & 2.03 & 0.06 & 2.60 & 0.07 & 2.11 & 0.09 & 1.51 & 0.20 \\
\hline & $\eta_{p}$ & 2.74 & 0.06 & 3.36 & 0.06 & 2.21 & 0.08 & 2.75 & 0.17 & 3.59 & 0.21 & 2.39 & 0.03 & 3.26 & 0.07 & 1.55 & 0.05 & 3.24 & 0.19 & 3.46 & 0.34 \\
\hline
\end{tabular}

Table 3

Summary of the Values of Solar Cycle Periodicity $T_{c}, e$-folding Time $\tau_{e}$ and Its Uncertainty $\delta$, and the Ratio $\tau_{e} / T_{c}$ for a Large Number of Configurations of the Triplet (Rm, $C_{\alpha}, C_{\mathrm{BL}}$ )

\begin{tabular}{|c|c|c|c|c|c|c|c|c|c|c|c|c|c|c|}
\hline & $C_{\mathrm{BL}}$ & $C_{\alpha}$ & $\mathrm{Rm}$ & $T_{c}$ & $\tau_{e}$ & $\delta$ & $\tau_{e} / T_{c}$ & $C_{\mathrm{BL}}$ & $C_{\alpha}$ & $\mathrm{Rm}$ & $T_{c}$ & $\tau_{e}$ & $\delta$ & $\tau_{e} / T_{c}$ \\
\hline & 0.50 & 16 & 308 & 9.02 & 24.48 & 3.50 & 2.71 & 1.00 & 16 & 368 & 9.40 & 42.15 & 5.50 & 4.48 \\
\hline & 0.50 & 16 & 318 & 12.78 & 23.25 & 5.94 & 1.81 & 1.00 & 16 & 378 & 9.28 & 46.00 & 17.59 & 4.95 \\
\hline & 0.50 & 16 & 328 & 12.52 & 23.33 & 6.79 & 1.86 & 1.00 & 20 & 318 & 9.73 & 23.74 & 4.77 & 2.43 \\
\hline & 0.50 & 16 & 338 & 12.13 & 25.40 & 5.61 & 2.09 & 1.00 & 24 & 308 & 9.48 & 20.98 & 5.80 & 2.21 \\
\hline & 0.50 & 16 & 348 & 11.61 & 26.37 & 5.74 & 2.29 & 1.00 & 24 & 318 & 9.47 & 19.00 & 2.72 & 2.00 \\
\hline & 0.50 & 16 & 358 & 11.49 & 29.06 & 6.61 & 2.52 & 1.00 & 24 & 328 & 9.26 & 19.15 & 3.67 & 2.06 \\
\hline & 0.50 & 16 & 368 & 11.26 & 26.60 & 3.29 & 2.36 & 1.00 & 24 & 338 & 9.94 & 20.85 & 4.02 & 2.09 \\
\hline & 0.50 & 16 & 378 & 11.04 & 42.66 & 2.52 & 3.86 & 1.00 & 24 & 348 & 8.96 & 21.75 & 1.67 & 2.42 \\
\hline & 0.75 & 16 & 318 & 10.64 & 28.05 & 4.62 & 2.63 & 1.00 & 24 & 358 & 8.84 & 19.74 & 2.81 & 2.23 \\
\hline & 1.00 & 8 & 308 & 11.13 & 32.21 & 5.67 & 2.89 & 1.00 & 24 & 368 & 8.61 & 25.07 & 4.39 & 2.91 \\
\hline \multirow[t]{9}{*}{$S$} & 1.00 & 8 & 318 & 10.95 & 36.64 & 4.41 & 3.34 & 1.00 & 24 & 378 & 8.52 & 21.06 & 2.48 & 2.47 \\
\hline & 1.00 & 8 & 328 & 10.77 & 62.13 & 14.35 & 5.76 & 1.00 & 28 & 318 & 9.32 & 18.54 & 2.00 & 1.99 \\
\hline & 1.00 & 8 & 338 & 10.50 & 52.30 & 9.98 & 4.98 & 1.00 & 32 & 318 & 9.28 & 16.75 & 2.46 & 1.80 \\
\hline & 1.00 & 8 & 348 & 10.30 & 47.97 & 7.21 & 4.65 & 1.25 & 16 & 318 & 10.19 & 33.69 & 10.99 & 3.30 \\
\hline & 1.00 & 8 & 358 & 10.17 & 53.44 & 19.78 & 5.25 & 1.50 & 16 & 308 & 10.13 & 25.21 & 2.01 & 2.48 \\
\hline & 1.00 & 8 & 368 & 10.08 & 63.59 & 3.03 & 6.30 & 1.50 & 16 & 318 & 10.30 & 24.12 & 2.57 & 2.34 \\
\hline & 1.00 & 8 & 378 & 10.07 & 54.52 & 3.27 & 5.41 & 1.50 & 16 & 328 & 10.14 & 25.50 & 3.35 & 2.51 \\
\hline & 1.00 & 12 & 318 & 10.53 & 32.81 & 3.36 & 3.11 & 1.50 & 16 & 338 & 9.45 & 22.46 & 7.07 & 2.37 \\
\hline & 1.00 & 16 & 308 & 10.18 & 27.35 & 2.36 & 2.68 & 1.50 & 16 & 348 & 9.56 & 25.77 & 4.00 & 2.69 \\
\hline \multirow[t]{5}{*}{$\mathrm{T}$} & 1.00 & 16 & 318 & 10.15 & 24.94 & 4.33 & 2.45 & 1.50 & 16 & 358 & 9.44 & 30.21 & 3.04 & 3.20 \\
\hline & 1.00 & 16 & 328 & 9.99 & 27.95 & 4.33 & 2.79 & 1.50 & 16 & 368 & 9.23 & 26.78 & 4.89 & 2.90 \\
\hline & 1.00 & 16 & 338 & 9.85 & 25.44 & 2.55 & 2.58 & 1.50 & 16 & 378 & 9.19 & 26.05 & 2.74 & 2.83 \\
\hline & 1.00 & 16 & 348 & 9.65 & 31.90 & 4.66 & 3.30 & 1.75 & 16 & 318 & 10.37 & 24.91 & 2.26 & 2.40 \\
\hline & 1.00 & 16 & 358 & 9.58 & 31.63 & 5.21 & 3.30 & 2.00 & 16 & 318 & 10.89 & 26.21 & 4.37 & 2.40 \\
\hline
\end{tabular}

Notes. The letters S and T make reference to the main two models discussed in the bulk of the paper. All the timescales are expressed in years. Boldface highlights the values of the timescales for the two specific cases ( $\mathrm{S}$ and $\mathrm{T}$ ) discussed throughout the text. 


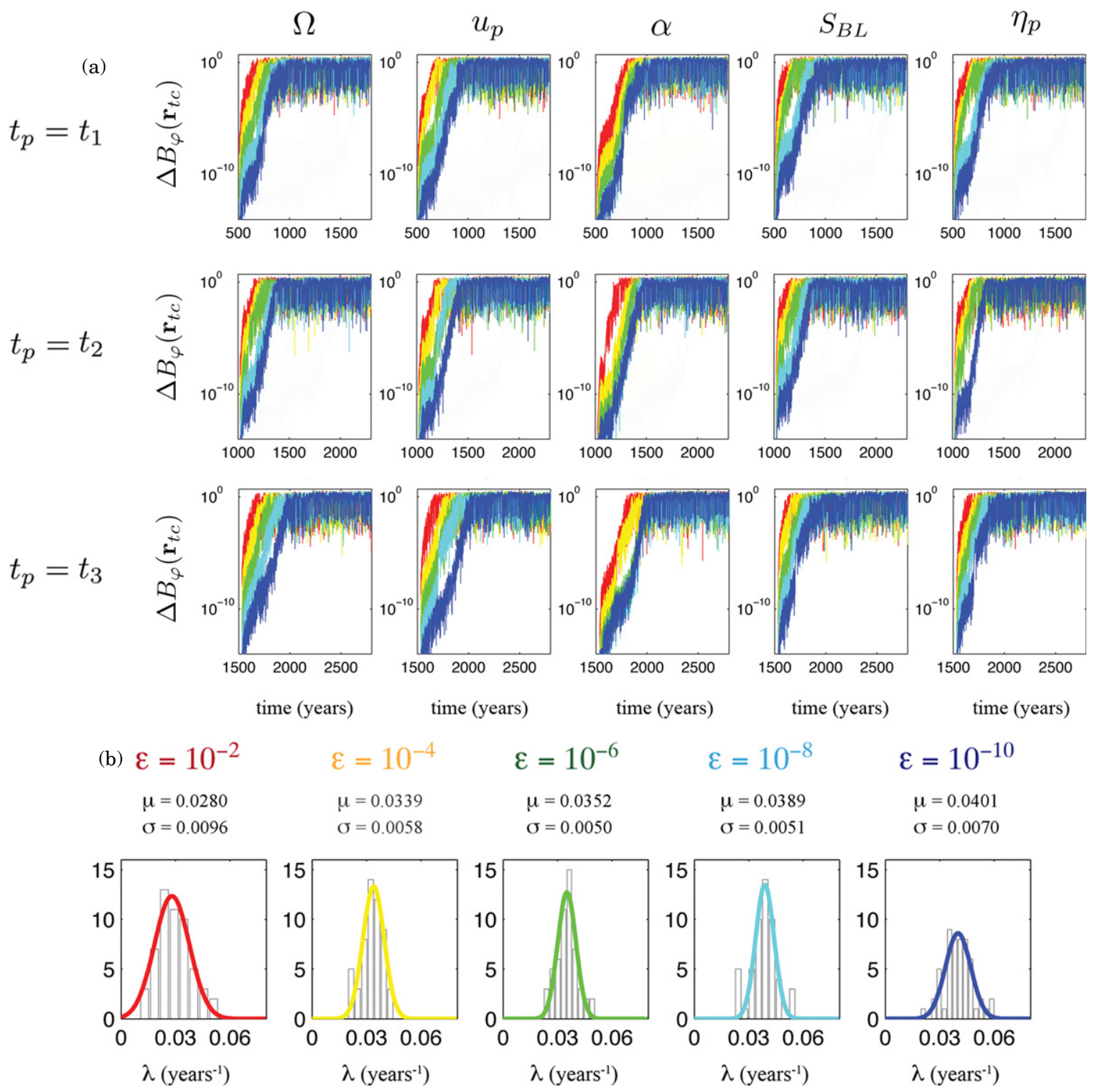

Figure 7. Same as Figure 6, for model T which has a stronger $\alpha$-effect than model S ( $C_{\alpha}=16$ instead of $\left.C_{\alpha}=8\right)$, all other control parameters being the same.

namely $10^{-10}$. This survey is summarized in Table 3 , and the results (expressed in terms of the corresponding timescales $T_{c}$ and $\tau_{e}$ ) are shown in Figures 8 and 9.

When $\mathrm{Rm}$ is fixed to its standard value (model $\mathrm{S}, \mathrm{Rm}=318$ ), our results show that $\tau_{e}$ and $T_{c}$ are mostly sensitive to $C_{\alpha}$, and less affected by variations in $C_{\mathrm{BL}}$. Regarding the former dependency, it can be seen in Figure 8(a) that both $\tau_{e}$ and $T_{c}$ decrease with $C_{\alpha}$. The decrease in $\tau_{e}$ is a consequence of the stronger destabilizing effect of turbulence. We also note (Figure 8(a), right) that the ratio $\tau_{e} / T_{c}$ decreases with increasing $C_{\alpha}$. In the parameter region which we explored, $\tau_{e}$ is thus more sensitive to variations in $C_{\alpha}$ than $T_{c}$.

According to Figure 8(b), the cycle period $T_{c}$ displays a non-monotonic behavior with respect to changes in $C_{\mathrm{BL}}$, which measures the intensity of the non-local coupling in the governing Equation (12). It is worth mentioning here that for the lower value of $C_{\mathrm{BL}}$, the system undergoes a transition to an $\alpha$ dominated dynamo, characterized by a longer (and less solarlike) periodicity of about $13 \mathrm{yr}$. As indicated by Figure 8(b), the $e$-folding time $\tau_{e}$ does not vary substantially with $C_{\mathrm{BL}}$ over our narrow interval of investigation (recall Section 3). Overall, we find that the ratio $\tau_{e} / T_{c}$ remains approximately constant (equal to 2.5) over this interval.

Turning our attention to the dependency of $T_{c}$ and $\tau_{e}$ on Rm, we see (Figure 9) that the former decreases with increasing $\mathrm{Rm}$. The cycle duration scales indeed approximately in inverse proportion to Rm, as shown in the left panel of Figure 9. On the other hand, the dependency of $\tau_{e}$ on Rm is less clear. There seems to be a mild trend in the cases of low to intermediate values of $C_{\alpha}$ (orange and red points in the middle plot of Figure 9), with $\tau_{e}$ slightly increasing with increasing $\mathrm{Rm}$. This behavior can be interpreted as a regulatory effect of the meridional circulation: as $u_{0}$ gets larger the meridional circulation tends to make the system more stable against perturbations. This is no longer true for a large $C_{\alpha}$ (dark red points in the middle plot of Figure 9), which indicates that $\tau_{e}$ is then controlled by the $\alpha$-effect. It is worth mentioning that some realizations of $\tau_{e}$ are affected by large uncertainties, mostly in cases with low values of $C_{\alpha}$ and $C_{\mathrm{BL}}$, and large values of $\mathrm{Rm}$. These cases are the less chaotic ones, and the introduction of a perturbation can sometimes lead to a mobilization phase lasting for more than $1000 \mathrm{yr}$. 

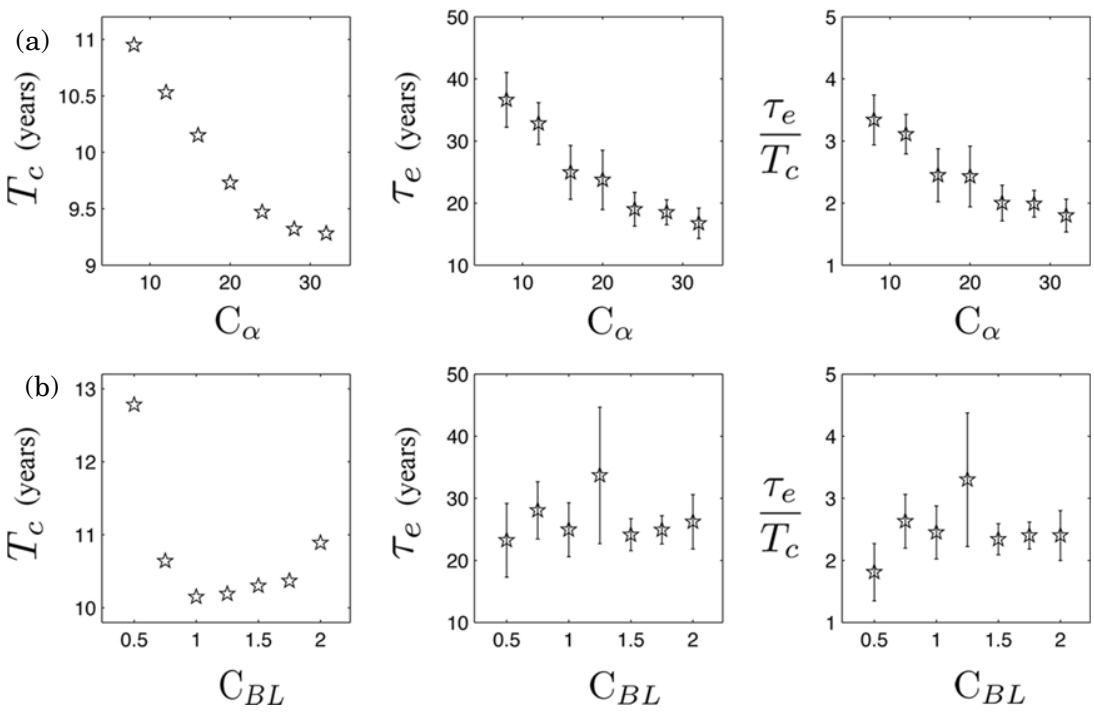

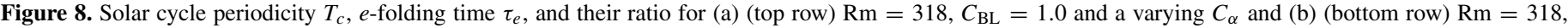
$C_{\alpha}=16$, and a varying $\mathrm{C}_{\mathrm{BL}}$.

To conclude this analysis, let us stress (as shown in Figure 9, right) that the $\tau_{e} / T_{c}$ ratio is mainly concentrated around two values, 2.5 and 5, with a larger concentration of points around the former. Using all the available data at our disposal (as summarized in Table 3), we can finally calculate a weighted average for the ratio $\tau_{e} / T_{c}$, and find

$$
\frac{\tau_{e}}{T_{c}}=2.76 \pm 0.05
$$

\section{SUMMARY AND DISCUSSION}

Our extensive analysis of the $e$-folding time $\tau_{e}$ for our preferred (in the sense of solar semblance) standard model $\mathrm{S}$ led us to conclude that if the control parameters $\left(\mathrm{Rm}, C_{\alpha}, C_{\mathrm{BL}}\right)$ are fixed, then $\tau_{e}$ can be regarded as an intrinsic property of the model, regardless of the source of the error, with a small dependence on its initial magnitude (Section 4.2.2).

In view of using that standard model (or a close version) for operational forecasting, we extended the analysis to a series of models, and investigated the sensitivity of $\tau_{e}$ to $\left(\mathrm{Rm}, C_{\alpha}, C_{\mathrm{BL}}\right)$ in detail. Our results reveal three salient properties.

1. A decrease of $\tau_{e}$ with increasing $C_{\alpha}$. This reflects the influence of the non-linear nature of the quenched $\alpha$-effect on the amplification of errors, leading to a more chaotic (and less predictable) dynamo.

2. An apparent independence of $\tau_{e}$ on $C_{\mathrm{BL}}$, indicating the secondary role played by this non-local forcing term on the error growth. However, let us stress that this may be caused by the narrow range of possible $C_{\mathrm{BL}}$ we explored, a consequence of the extreme sensitivity of the solar semblance of the flux-transport model to this parameter.

3. A slight tendency for $\tau_{e}$ to increase with $\mathrm{Rm}$ for those models with low to intermediate strength of the $\alpha$-effect, pointing to a stabilizing role of the meridional circulation on the system under these conditions.

In addition, the moderate variability of the ratio of $\tau_{e}$ to the simulated cycle period $T_{c}$ in our database of simulations (which comprises approximately 50 members) prompts us to propose the master value $\bar{\tau}_{e}=2.76 T_{c}$ for the class of mean-field models we considered, should they be used for operational forecasting (and keeping in mind that we focused our analysis on the regular working of those models, not considering extreme events such as grand minima).

From a practical point of view, the perturbations artificially inserted into the model in Section 4 can be interpreted as uncertainties in the measurements or in the model itself, which are the causes of errors any data assimilation scheme needs to deal with. These uncertainties are ultimately responsible for the limited horizon of predictability of the chaotic system we are interested in. If $\varepsilon$ denotes the relative level of these uncertainties, we derive from Equation (29) that the forecast horizon $\tau_{f}$ is given by

$$
\tau_{f}=-\bar{\tau}_{e} \ln \varepsilon
$$

Let us begin by estimating the level of uncertainties on the measurement side. It is likely that an operational data assimilation scheme will assimilate observations connected with largescale maps of $B_{r}$ at the solar surface, $B_{r}^{S}$. Such magnetograms are contaminated by errors, due to limited resolution, asynchronous sampling and sparse polar measurements. A way to quantify those errors is to analyze the spherical harmonic decomposition of $B_{r}^{S}$. Theory demands the monopole term in this expansion $\left(g_{0}^{0}\right)$ to be zero; a non-zero $g_{0}^{0}$ can consequently be used as a means to quantify the uncertainty $\varepsilon\left(B_{r}^{S}\right)$ we are after. Figure 10 shows the time series of the monopole and axial dipole coefficient $\left(g_{1}^{0}\right)$ derived from the database of magnetograms of the Wilcox Solar Observatory (WSO). ${ }^{1}$ The figure shows that $g_{1}^{0}$ evolves in phase with the global poloidal magnetic field-it changes sign at the time of maximum activity, and is correlated with the polar flux (DeRosa et al. 2012). The monopole coefficient $g_{0}^{0}$ constantly oscillates around zero. We can therefore use the ratio of the root-mean-squared (rms) value of $g_{0}^{0},\left\langle g_{0}^{0}\right\rangle$, to the rms value of $g_{1}^{0},\left\langle g_{1}^{0}\right\rangle$, to estimate $\varepsilon\left(B_{r}^{S}\right)$. This yields

$$
\varepsilon\left(B_{r}^{S}\right) \approx \frac{\left\langle g_{0}^{0}\right\rangle}{\left\langle g_{1}^{0}\right\rangle}=\frac{0.1535 \mathrm{G}}{1.2550 \mathrm{G}} \approx 12 \% .
$$

On the model side now, one of the most obvious sources of errors lies in the large-scale kinematic approximation on which

\footnotetext{
1 http://wso.standford.edu/Harmonic.rad/ghlist.html
} 

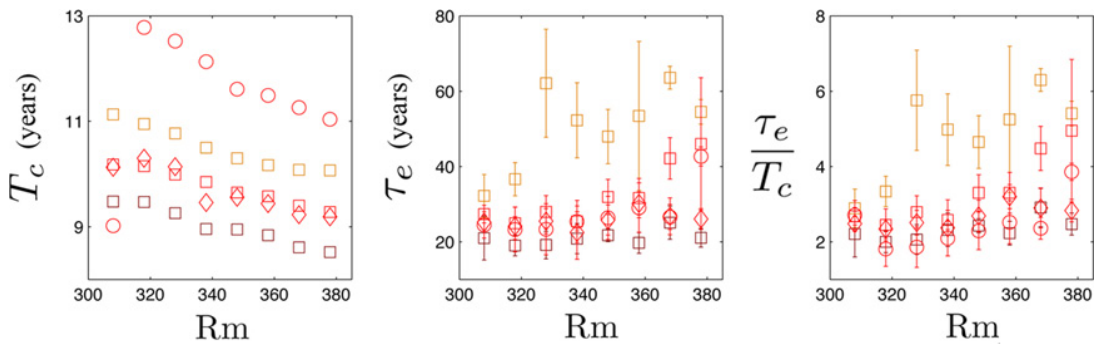

Figure 9. Solar cycle periodicity $T_{c}, e$-folding time $\tau_{e}$ and their ratio for different values of $C_{\alpha}, C_{\mathrm{BL}}$ and Rm. The magnitude of $C_{\alpha}$ is color-coded: $C_{\alpha}=8$, orange; $C_{\alpha}=16$, red; $C_{\alpha}=24$, dark red. Symbols indicate different $C_{\mathrm{BL}}: C_{\mathrm{BL}}=0.5$, circles; $C_{\mathrm{BL}}=1.0$, squares; $C_{\mathrm{BL}}=1.5$, diamonds

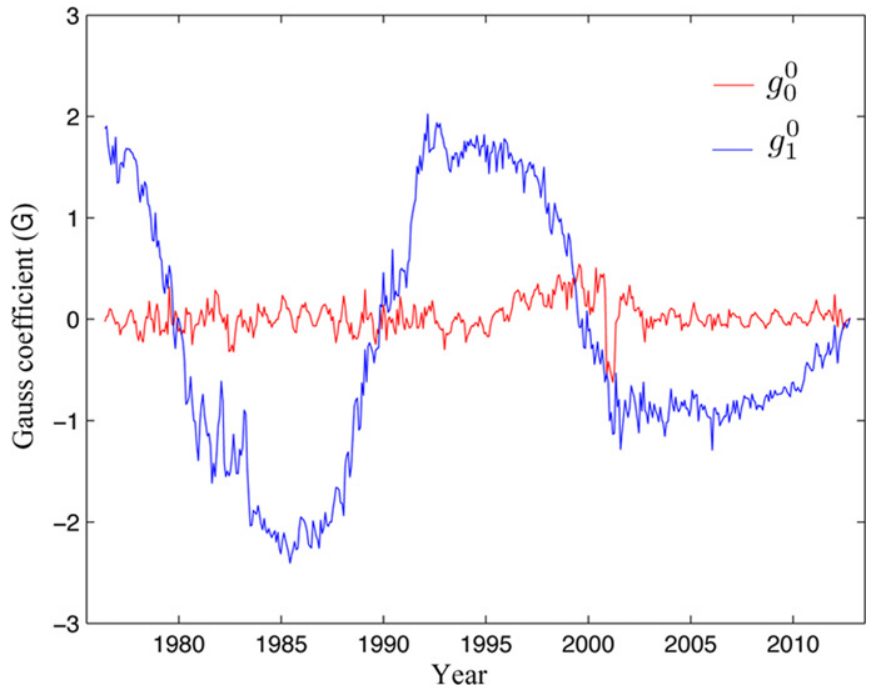

Figure 10. Time series of the monopole $g_{0}^{0}$ and axial dipole $g_{1}^{0}$ Gauss coefficients derived from the magnetic charts of the Wilcox Solar Observatory (http://wso.standford.edu/Harmonic.rad/ghlist.html).

our modeling rests. In particular, observations indicate that it may be inappropriate to assume that the large-scale flow driving the dynamo is steady. As a consequence, there are errors arising from the variability of both the patterns of differential rotation and meridional circulation. Howe et al. (2000) discovered a persistent pattern of low-amplitude time variation of $\Omega, \delta \Omega$, of about $6 \mathrm{nHz}$, due to solar torsional oscillations. Consequently, we get

$$
\varepsilon(\Omega)=\frac{\delta \Omega}{\Omega_{\mathrm{eq}}} \approx 1 \% .
$$

This small figure must be contrasted with the one owing to those uncertainties impacting $\mathbf{u}_{p}$. The long-term variability of the meridional circulation $\delta \mathbf{u}_{p}$ has an amplitude $\delta u_{0}$ close to $5 \mathrm{~m} \mathrm{~s}^{-1}$ (Hernández et al. 2006), which yields

$$
\varepsilon\left(\mathbf{u}_{p}\right)=\frac{\delta u_{0}}{u_{0}} \approx 33 \%
$$

if computed based on the mean value of the surface meridional flow at mid-latitudes, $u_{0} \approx 15 \mathrm{~m} \mathrm{~s}^{-1}$. Injecting $\varepsilon\left(\mathbf{u}_{p}\right)$ in Equation (33) yields

$$
\tau_{f} \approx 3 T_{c}
$$

In addition to these fluctuations in amplitude, there exists considerable uncertainties on the large-scale structure of the meridional circulation itself. The depth at which the equatorward return flow occurs (Hathaway 2011) and the possible multi-cell pattern of meridional flow (Zhao et al. 2013) are two examples illustrating the current lack of robust observational constraints on $\mathbf{u}_{p}$. These cannot be readily incorporated in the current analysis, for they would require different families of simulations to be integrated, and their region of solar semblance be identified in parameter space (in the same way we mapped it for the ensemble of single-cell, tachocline equatorward return flow simulations considered here).

Further uncertainties affect the turbulent diffusivity, $\eta(\mathbf{r})$. As explained in Section 2, we resort in this study to spherically symmetric $\eta_{p}(r)$ and $\eta_{t}(r)$, of relatively low values. Both reach an amplitude of $3 \times 10^{11} \mathrm{~cm} \mathrm{~s}^{-2}$ at $r=R_{\odot}$ (recall Figure 2(d)). This value has to be contrasted with the value of $10^{12} \mathrm{~cm} \mathrm{~s}^{-2}$ used by Choudhuri et al. (2007) for their prediction of cycle 24, using a so-called diffusion-dominated flux-transport model. More recently, Miesch et al. (2012) put forward theoretical and observational arguments in favor of the same figure, $10^{12} \mathrm{~cm} \mathrm{~s}^{-2}$, as a lower bound of this turbulent transport coefficient. The exact nature of $\eta(\mathbf{r})$ in the solar interior remains strongly debated, and no consensus has been reached.

In this study, we opted for an advection-dominated forward model, on the account of its first order dynamical semblance with the solar dynamo. If one were to choose instead a diffusiondominated model for data assimilation purposes, one would have to carry out a sensitivity analysis similar to the one pursued here, in order to compute the $e$-folding time and estimate the forecast horizon for that different family of models. In this respect, note that Karak \& Nandy (2012) resorted to a simpler, correlation-based, analysis in order to study the "memory" of such a diffusion-dominated model (which includes in their case turbulent pumping and a stochastic component to the poloidal source term). Their analysis demonstrates that turbulent diffusion shortens the memory of the system to less than one cycle. This preliminary work should be complemented by the proper derivation of the $e$-folding time characterizing that class of models, along the methodological lines presented in this paper.

Regardless of the model ultimately chosen, one should keep in mind that data assimilation remains in any case the only sensible way of testing the compatibility of a given physical model of the solar dynamo with observations of its dynamical activity. By enabling on-the-fly parameter adjustments (in addition to state estimation), data assimilation offers in principle the possibility of correcting the radial profiles of diffusion coefficients (and those of poloidal source terms). Even if the advection-dominated model we studied has an optimistic theoretical predictability limit of three solar cycles, we must bear in mind that any data assimilation scheme aiming to forecast solar activity will be unperfect, and its effective forecast 

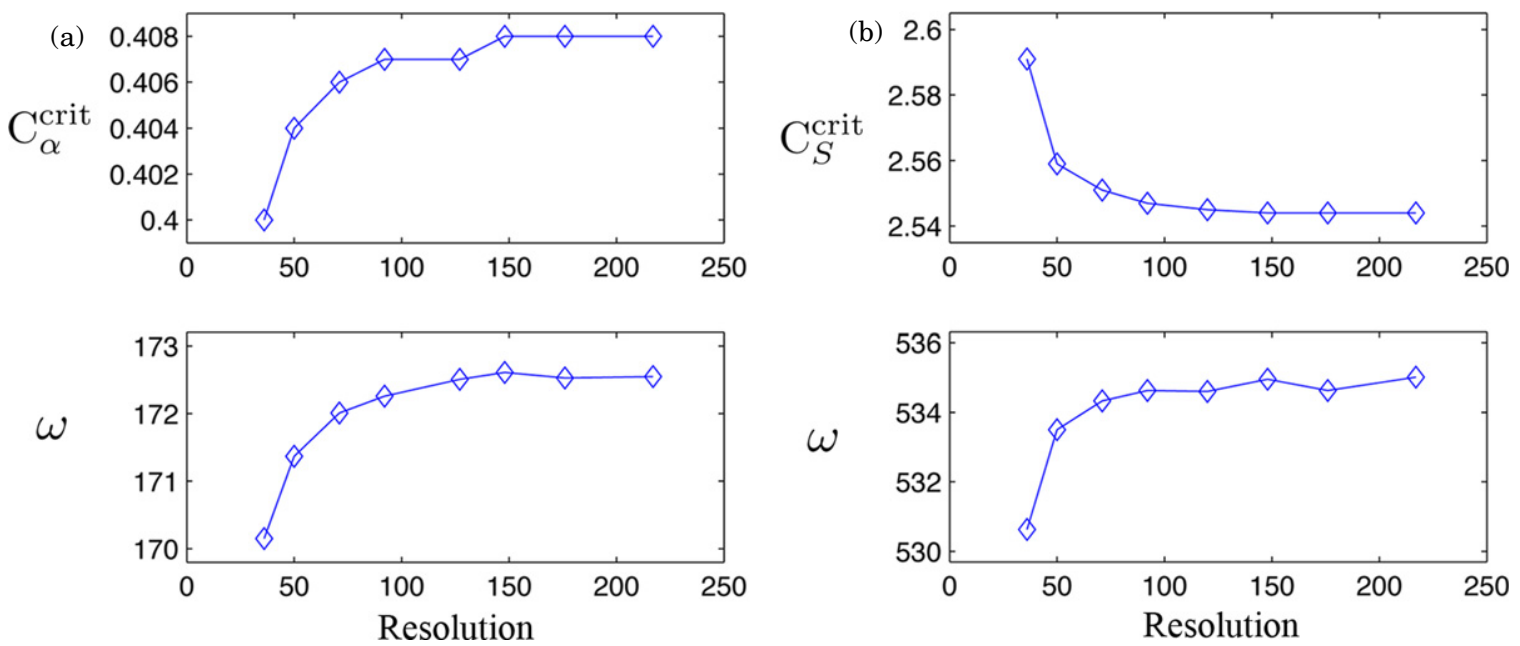

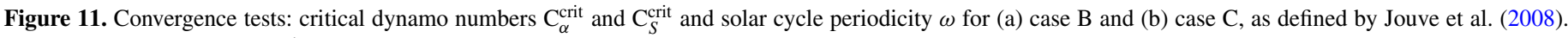
The resolution is defined by $\sqrt{N_{r} N}$, where $N_{r}$ is the number of radial levels and $N$ is the truncation of the spherical harmonic expansion.

(A color version of this figure is available in the online journal.)

Table 4

Comparison of the Critical Dynamo Numbers $C_{\alpha, S}^{\text {crit }}$ and Frequency of the Solar Cycle $\omega$ in the Benchmark Cases A, B and C from Jouve et al. (2008)

\begin{tabular}{lcccccrr}
\hline \hline Case & \multicolumn{4}{c}{ Results } & & & \multicolumn{2}{c}{ Reference } \\
\cline { 2 - 4 } & Resolution & $\Delta t$ & $C_{\alpha, s}^{\text {crit }}$ & $\omega$ & & $C_{\alpha, s}^{\text {crit }}$ & \multicolumn{1}{c}{$\omega$} \\
\hline A & $71 \times 71$ & $5 \times 10^{-5}$ & 0.385 & 158.00 & & $0.387 \pm 0.002$ & $158.1 \pm 1.472$ \\
B & $71 \times 71$ & $5 \times 10^{-5}$ & 0.406 & 172.01 & & $0.408 \pm 0.003$ & $172.0 \pm 0.632$ \\
C & $120 \times 120$ & $1 \times 10^{-6}$ & 2.545 & 534.6 & & $2.489 \pm 0.075$ & $536.6 \pm 8.295$ \\
\hline
\end{tabular}

Notes. The spacial and temporal resolutions are given in terms of radial points and harmonic degree $\left(N_{r} \times N\right)$ and time-step $\Delta t$.

horizon will consequently decrease. Taking this into account, one can hope, though, that if such an advection-dominated model were to be chosen for operational forecasting, its practical limit of predictability could reach (and perhaps exceed) one solar cycle.

The authors thank the referee for helpful and constructive review, and Allan Sacha Brun, Emmanuel Dormy and Martin Schrinner for enlightening discussions. Sabrina Sanchez also thanks Oscar Matsuura and Katia Pinheiro for the fruitful contributions to the beginning of this project, the Observatório Nacional of Brazil for the initial support, and the Space Physics and Aeronomy group of the American Geophysical Union for the student grant award at the 2012 AGU Meeting. Numerical calculations were performed on IPGP's S-CAPAD computing facility. This is IPGP contribution 3459.

\section{APPENDIX}

\section{PARODY CODE-MEAN FIELD BENCHMARKING}

The Parody code used in this work was originally proposed for full 3D MHD dynamo simulations (ACD code, benchmarked in Christensen et al. 2001; see Dormy et al. 1998 and Aubert et al. 2008). In order to perform an analysis of the predictability of standard mean-field solar dynamos, it was necessary to ensure the compatibility of the model with the ones used within the solar dynamo community. For such reason, we modified and compared outputs from our 3D MHD code with a mean-field solar dynamo benchmark.
The full spherical harmonic expansion of the code writes

$$
(\mathcal{P}, \mathcal{T})(r, \theta, \varphi, t)=\sum_{n=1}^{N} \sum_{m=1}^{M}\left(\mathcal{P}_{n}^{m}, \mathcal{T}_{n}^{m}\right)(r, t) Y_{n}^{m}(\theta, \varphi)
$$

truncated at spherical harmonic degree and order $N$ and $M$, respectively. As most mean-field models assume axisymmetry, we set $M=0$ throughout.

The original inner boundary conditions of Parody considered the inner core as an insulating or electrically conducting medium of finite conductivity (Christensen et al. 2001); in contrast, in the solar context, the radiative zone is modeled as a perfect conductor. This last condition requires to impose

$$
\begin{gathered}
\mathcal{P}=0, \text { and } \\
\partial(r \mathcal{T}) / \partial r=0 \text { at the inner boundary. }
\end{gathered}
$$

Further modifications of the code included the incorporation of the $\alpha$ and BL source terms in the poloidal induction Equation (12), and the prescription of the flow fields, $\Omega$ and $\mathbf{u}_{p}$, and depth-dependent turbulent diffusivities $\eta(r)$.

The resulting code was tested against published reference solutions of the mean-field community benchmark effort described by Jouve et al. (2008). The benchmarking consists of computing the critical dynamo numbers $C^{\text {crit }}$, and solar activity cycle frequency $\omega$, for three case studies. The three cases include two $\alpha \Omega$ mean-field dynamos (cases $\mathrm{A}$ and $\mathrm{B}$, differing only by the prescribed $\eta(r)$ ) and a BL dynamo (case C). Table 4 displays the values obtained from our code and the Jouve et al. (2008) benchmark ones for each case, while convergence tests 

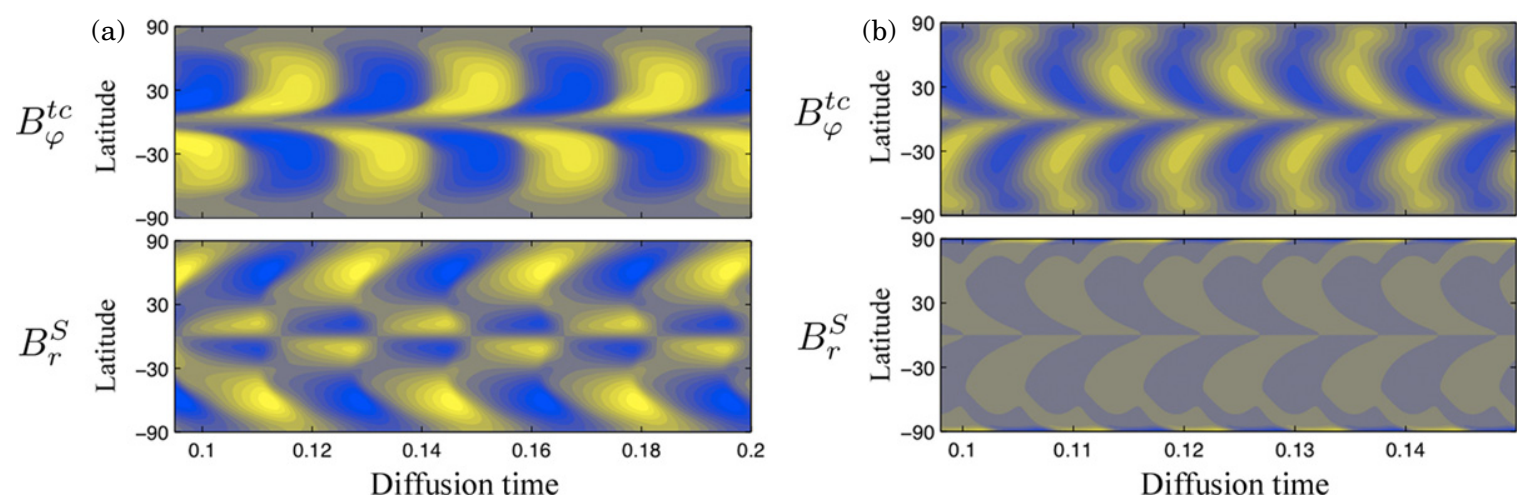

Figure 12. Butterfly diagrams summarizing two different benchmark cases from Jouve et al. (2008): (a) $\alpha \Omega$ dynamo from the supercritical case SB and (b) a Babcock-Leighton dynamo from the supercritical case SC. For each case, the upper panel displays the toroidal field at the tachocline and the lower one the radial field at the surface.

(A color version of this figure is available in the online journal.)

of the critical numbers of cases B and C are shown in Figure 11. In addition, butterfly diagrams for the supercritical cases SB and SC (the supercritical cases include $\alpha$ and $S_{\mathrm{BL}}$ quenching) are displayed in Figure 12.

Note that in the present study, and compared with the benchmark cases, we use slightly different inner boundary conditions, namely

$$
\begin{gathered}
\mathcal{P}=0 \text {, and } \\
\mathcal{T}=0 \text { at the inner boundary, }
\end{gathered}
$$

as is common in mean-field solar dynamo simulations (e.g., Dikpati \& Charbonneau 1999). Inspection of results obtained with both types of inner boundary conditions shows that they are virtually the same, in agreement with Chatterjee et al. (2004). An interpretation of this is that the low diffusivity of the radiative zone and the absence of a deeply penetrating meridional flow inhibit the penetration of the strong tachocline magnetic field to the deepermost layers.

\section{REFERENCES}

Aubert, J., Aurnou, J., \& Wicht, J. 2008, GeoJI, 172, 945

Aubert, J., \& Fournier, A. 2011, NPGeo, 18, 657

Babcock, H. W. 1961, ApJ, 133, 572

Baker, D. N. 2000, ITPS, 28, 2007

Bonanno, A., Elstner, D., Rüdiger, G., \& Belvedere, G. 2002, A\&A, 390,673

Brasseur, P. 2006, in Ocean Weather Forecasting: An Integrated View of Oceanography, ed. E. Chassignet \& J. Verron (Dordrecht, The Netherlands: Springer), 271

Brun, A. S. 2007, AN, 328, 329

Brun, A. S. M. S., \& Toomre, J. 2004, ApJ, 614, 1073

Bushby, P. J., \& Tobias, S. M. 2007, ApJ, 661, 1289

Charbonneau, P. 2005, LRSP, 2, 2

Charbonneau, P., \& Dikpati, M. 2000, ApJ, 543, 1027

Charbonneau, P., \& Smolarkiewicz, P. K. 2013, Sci, 340, 42

Charbonneau, P., St-Jean, C., \& Zacharias, P. 2005, ApJ, 619, 613

Chatterjee, P., Nandy, D., \& Choudhuri, A. R. 2004, A\&A, 427, 1019

Choudhuri, A. R., Chatterjee, P., \& Jiang, J. 2007, PhRvL, 98, 131103

Christensen, U. R., Aubert, J., Cardin, P., et al. 2001, PEPI, 128, 25

DeRosa, M. L., Brun, A. S., \& Hoeksema, J. T. 2012, ApJ, 757, 96

Dikpati, M., \& Anderson, J. L. 2012, ApJ, 756, 20
Dikpati, M., \& Charbonneau, P. 1999, ApJ, 518, 508

Dikpati, M., De Toma, G., \& Gilman, P. A. 2006, GeoRL, 33, L05102

Dikpati, M., \& Gilman, P. A. 2001, ApJ, 559, 428

Dormy, E., Cardin, P., \& Jault, D. 1998, E\&PSL, 160, 15

D'Silva, S., \& Choudhuri, A. R. 1993, A\&A, 272, 621

Elbern, H., Strunk, A., \& Nieradzik, L. 2010, in Data Assimilation, ed. W. Lahoz, B. Khattatov, \& R. Menard (Berlin: Springer), 491

Fan, Y. 2009, LRSP, 6, 4

Fournier, A., Eymin, C., \& Alboussière, T. 2007, NPGeo, 14, 163

Fournier, A., Hulot, G., Jault, D., et al. 2010, SSRv, 155, 247

Fournier, A., Nerger, L., \& Aubert, J. 2013, GGG, 14, 4035

Haigh, J. D. 2003, RSPTA, 361, 95

Hathaway, D. H. 1996, ApJ, 460, 1027

Hathaway, D. H. 2009, SSRv, 144, 401

Hathaway, D. H. 2010, LRSP, 7, 1

Hathaway, D. H. 2011, arXiv:1103.1561

Hernández, I. G., Komm, R., Hill, F., et al. 2006, ApJ, 638, 576

Houser, P. R., De Lannoy, G. J., \& Walker, J. P. 2010, in Data Assimilation, ed. W. Lahoz, B. Khattatov, \& R. Menard (Berlin: Springer), 549

Howe, R., Christensen-Dalsgaard, J., Hill, F., et al. 2000, Sci, 287, 2456

Hulot, G., Lhuillier, F., \& Aubert, J. 2010, GeoRL, 37, 6

Jouve, L., Brun, A. S., Arlt, R., et al. 2008, A\&A, 483, 949

Jouve, L., Brun, A. S., \& Talagrand, O. 2011, ApJ, 735, 31

Jouve, L., Proctor, M. R. E., \& Lesur, G. 2010, A\&A, 519, 13

Kalnay, E. 2003, Atmospheric Modeling, Data Assimilation and Predictability (Cambridge: Cambridge Univ. Press)

Karak, B. B., \& Nandy, D. 2012, ApJL, 761, L13

Kitiashvili, I., \& Kosovichev, A. G. 2008, ApJL, 688, L49

Leighton, R. B. 1969, ApJ, 156, 1

Lhuillier, F., Aubert, J., \& Hulot, G. 2011, GeoJI, 186, 492

Lorenz, E. N. 1963, JAtS, 20, 130

Lorenz, E. N. 1965, Tell, 17, 321

Miesch, M. S., Featherstone, N. A., Rempel, M., \& Trampedach, R. 2012, ApJ, 757, 128

Moffatt, H. K. 1978, Field Generation in Electrically Conducting Fluids (Cambridge: Cambridge Univ. Press)

Ossendrijver, M. 2003, A\&ARv, 11, 287

Parker, E. N. 1955, ApJ, 122, 293

Priest, E. R. 1982, Solar Magnetohydrodynamics (Dordrecht: Reidel)

Pulkkinen, T. 2007, LRSP, 4, 1

Sanchez, S. M., Fournier, A., Pinheiro, K. J. R., \& Aubert, J. 2014, An. Acad. Bras. Cienc., 86, in press (arXiv:1307.4115)

Talagrand, O. 1997, MeSJJ, 75, 81

Tomczyk, S., Schou, J., \& Thompson, M. J. 1995, ApJL, 448, L57

Vallis, G. K. 2006, Atmospheric and Oceanic Fluid Dynamics: Fundamentals and Large-scale Circulation (Cambridge: Cambridge Univ. Press)

Wang, Y. M., \& Sheeley, N. R. 2009, ApJL, 694, L11

Zhao, J., Bogart, R., Kosovichev, A., Duvall, T., Jr., \& Hartlep, T. 2013, ApJL, 774, L29 